\title{
Hausdorff separability of the boundaries for spacetimes and sequential spaces.
}

\author{
J.L. Flores* , J. Herrera ${ }^{\ddagger}$, M. Sánchez ${ }^{\dagger}$ \\ * Departamento de Álgebra, Geometría y Topología, \\ Facultad de Ciencias, Universidad de Málaga, \\ Campus Teatinos, 29071 Málaga, Spain \\ ${ }^{\ddagger}$ Instituto de Matemática e Estatística, \\ Universidade de São Paulo, \\ Rua do Matão, 1010, Cidade Universitaria São Paulo, Brazil \\ ${ }^{\dagger}$ Departamento de Geometría y Topología, \\ Facultad de Ciencias, Universidad de Granada, \\ Avenida Fuentenueva s/n, 18071 Granada, Spain \\ January 6, 2015
}

There are several ideal boundaries and completions in General Relativity sharing the topological property of being sequential, i.e., determined by the convergence of its sequences and, so, by some limit operator $L$. As emphasized in a classical article by Geroch, Liang and Wald, some of them have the property, commonly regarded as a drawback, that there are points of the spacetime $M$ non $T_{1}$-separated from points of the boundary $\partial M$.

Here we show that this problem can be solved from a general topological viewpoint. In particular, there is a canonical minimum refinement of the topology in the completion $\bar{M}$ which $\mathrm{T}_{2}$-separates the spacetime $M$ and its boundary $\partial M$-no matter the type of completion one chooses. Moreover, we analyze the case of sequential spaces and show how the refined $T_{2}$-separating topology can be constructed from a modification $L^{*}$ of the original limit operator $L$. Finally, we particularize this procedure to the case of the causal boundary and show how the separability of $M$ and $\partial M$ can be introduced as an abstract axiom in its definition.

Keywords: boundaries of spacetimes, causal, conformal, geodesic and bundle boundary, Hausdorff separability, sequential space, limit operator.

MSC: Primary 53C50, 83C75, Secondary: 54D55, 54A20. 


\section{Contents}

1 Introduction 2

2 Limit operators and sequential spaces 4

2.1 General properties . . . . . . . . . . . . . . . . 4

2.2 Higher order limit operators . . . . . . . . . . . . . . . . . . 7

$3 \mathbf{T}_{2}$-separating boundaries in the general and sequential cases 8

3.1 Separating for an arbitrary topology . . . . . . . . . . . . . 8

3.2 Separating among sequential topologies . . . . . . . . . . . 9

3.3 Limit operators and further properties of the separating topology . . . . . . . . 10

4 Completions of spacetimes, $T_{2}$-separability and c-boundary 14

4.1 A revision of some boundaries in Relativity . . . . . . . . . . . . . . . . 14

4.2 Basics on the c-boundary . . . . . . . . . . . . . . . . 15

4.3 The modified c-boundary . . . . . . . . . . . . . . . . . 18

5 Admissibility criteria for c-boundaries 19

5.1 Original criteria . . . . . . . . . . . . . . . . . . . 19

5.2 Including $D$-separability as an admissibility condition . . . . . . . . . . 21

6 Appendix: some examples 22

\section{Introduction}

A recurrent topic in the mathematical study of relativistic spacetimes, is the definition of some types of ideal boundaries which encode relevant information about them. Being this a natural practice from a mathematical viewpoint, there are further physical motivations coming from the holographic principle and the anti-de Sitter/conformal field theory correspondence. There have been quite a few of proposals of boundaries (including geodesic, bundle, abstract, conformal and causal ones, see Section 4), each one with its own limitations. One of the most common problems appears from the topological viewpoint, as typically one may find that some point of the spacetime $M$ is not topologically well separated (i.e., non-Hausdorff separated or even non$\mathrm{T}_{1}$-separated) from some point of the boundary $\partial M$. Such a property seems so undesirable, that has been considered as a reason to reject a priori many possible boundaries [15]. The purpose of this paper is to reconsider this question from a broad mathematical perspective.

In a naive approach to the problem, one would like that a boundary $\partial M$ satisfied: (i) it inherits the good topological properties of the topology of the spacetime $M$, and (ii) it records information of missing points, that is, it would satisfy, for example: if a point of the spacetime is removed, the boundary will identify this unequivocally and will allow to restore the missing point. However, a closer look shows that these requirements would be too restrictive. About the requisite (i), notice first that one can find an open subset $D$ of Euclidean space $\mathbb{R}^{n}$ with, say, a fractal topological boundary $\partial D \subset \mathbb{R}^{n}$. A worse contradiction with (i) appears when some of the commented relativistic boundaries yield naturally a couple of non $\mathrm{T}_{2}$-separated boundary points: this becomes a natural consequence of the fact that there is no any distance associated to a spacetime ${ }^{1}$. About (ii), assume that one is considering a boundary invariant by homotheties.

${ }^{1}$ When a distance exists on $M$ (as happens, for example, when $M$ is endowed with a -positive definite- Riemannian metric) one may expect that it will be extensible to the boundary $\partial M$ and, thus, $\partial M$ would be Hausdorff. 
Then one cannot distinguish between removing a point and removing a closed ball in Euclidean space, that is, between $\mathbb{R}^{n} \backslash\{0\}$ and $\mathbb{R}^{n} \backslash \bar{B}(0,1)$. Thus, it would not be clear if such a boundary should restore either a point or a sphere. This shows that (ii) may be also too restrictive; for example, it cannot be expected for any conformally invariant boundary, as some of the relativistic ones.

Notice that, in certain sense, the bad topological separation between some $p \in M$ and some $Q \in \partial M$, appears naturally when the requirements (i) and (ii) fail: by the failure of the former, one can admit the bad topological separation of two boundary points $P, Q \in \partial M$ but, because of the failure of the latter, one might discover that $P$ admits an interpretation as a missing point $p$ for some new spacetime. Roughly, our answer to this problem will be the following:

No matter how the completion $\bar{M}=M \cup \partial M$ is constructed, one can define a unique minimal refinement of the topology (i.e. a finer topology) in $\bar{M}$ which will $T_{2}$-separate the points of the manifold $M$ and those of the boundary $\partial M$. So (whenever the spacetime $M$ is clearly identified in the completion $\bar{M}$ ), this refined completion would not be rejected a priori by claiming pathological separability properties. Moreover, the minimal character of the refinement will preserve most of the desirable properties of the original topology.

Once this aim is carried out, our next aim is motivated by the following observation. Some of the previous topologies are defined in terms of a limit operator $L$ which characterizes the convergence of the sequences. Thus, it is interesting to study especially this case and, concretely, how to construct a Hausdorff separating topology by modifying directly L. Mathematically, the spaces whose topology can be characterized by such an operator are the sequential spaces. This is a vast class of spaces (it includes all the first countable ones, in particular the metric spaces, see Section 3). So, we will also carry out a study of independent mathematical interest about the refinement process for sequential spaces endowed with a limit operator.

As a last aim, this study will be applied specifically to the causal boundary (c-boundary for short in the remainder) of spacetimes. The reason is twofold. On the one hand, this boundary has been widely studied recently, because it seems the unique intrinsic and general alternative to the Penrose conformal boundary (the latter is commonly used in Mathematical Relativity, but its existence is ensured only in quite particular cases). On the other hand, the redefinition of the c-boundary in [9] left open the possibility of new refinements of such a notion, by imposing additional properties to the boundary which might be judged as desirable a priori. The property of separation studied here is a good example of this possibility.

This article is organized as follows. In Section 2 we recall some general features of sequential spaces, and prove some first properties about limit operators (Subsection 2.1. specially Propositions 2.5 and 2.7). The following subtlety is emphasized. A limit operator $L$ for a topology $\tau$ is full or of first order when $L$ provides directly all the limits of all the convergent sequences for $\tau$; in this case, it is univocally determined and can be denoted by $L_{\tau}$. Otherwise, $L$ determines only some limits of convergent sequences, which are enough to determine the topology. Even more, one can wonder if $L$ is of second order (roughly, the iteration of $L$ twice is enough to determine $L_{\tau}$ ) or of other higher orders; this is briefly explained in Subsection 2.2. Even though any topology (sequential or not) admits a unique first order $L_{\tau}$, it is interesting to consider also the case of non-first order limit operators because: (a) the topology of some relativistic boundaries is defined by imposing that naturally, some sequences must converge; however, such a convergence

But recall that even a non-symmetric distance, as the one associated to a non-reversible Finsler metric, may lose properties when extended to the boundary. In fact, the Cauchy completion of a Finsler manifold may not be $T_{1}$, see [11 Section 3]. This is relevant for the case of spacetimes too, as Finsler metrics appear naturally when the conformal class of simple classes of spacetimes are considered [6] 11]. 
may imply some other (possibly undesired) limits, and (b) in principle, simple modifications of $L$ (as, eventually, those required for our refinement of the topologies) might give limit operators that are not of first order, even if $L$ was.

Section 3. In Subsection 3.1. the problem of $T_{2}$-separating a domain $D$ of its complement $X \backslash D$, in an arbitrary topological space $(X, \tau)$ (see Defn. 3.1), is considered. A (minimum) unique explicit topology $\tau^{*}$ is shown to solve the problem (Theorem 3.3). In Subsection 3.2 , we consider the problem for the special case of sequential spaces. Recall that for these spaces, it is natural to wonder about a separating topology which is minimum among the sequential ones. The existence of a unique sequential topology $\tau_{S e q}^{*}$ solving this problem of $T_{2}$-separation is also obtained (Theorem 3.5). Finally, in Subsection 3.3 we analyze the role of the chosen limit operator $L$ for the case of sequential topologies. Concretely, the domain $D$ allows to define naturally a modification $L^{*}$ of the original operator $L$ (see formula (8)). When $L$ is of first order, the topology generated by $L^{*}$ can be identified with the topology previously obtained (see Theorem 3.9 and Figure 1. notice that $L^{*}$ is then necessarily of first order too); otherwise, some other properties still hold (Proposition 3.7).

Section 4. In Subsection 4.1 we review briefly the most common boundaries for spacetimes, and explain how previous results can be applied to ensure the Hausdorff separability between points of $M$ and $\partial M$. Special care is put in the discussion of a general example by Geroch, Liang and Wald [15] which shows that, under general hypotheses, the g-boundary and other boundaries introduced in General Relativity will have a pair of non- $T_{1}$ related points, one of them in the spacetime and the other in the boundary. Our previous results allow to circumvent this problem and suggest that the g-boundary should be redefined. In the remainder we focus in the case of the c-boundary, whose topology (the chronological topology $\tau_{c h r}$ ) is defined as the one derived from some limit operator $L_{c h r}$. In Subsection 4.2, a short review of the causal completion is provided. In Subsection 4.3 we check that the good properties of this completion (previously summarized in Theorem 4.3) are maintained when one uses both, the corresponding separating topology $\tau_{c h r}^{*}$ and the sequentially separating one $\left(\tau_{c h r}^{*}\right)_{S e q}$ (Theorem 4.4.

Section 5. Here, we analyze the process to define the c-boundary from very general admissibility properties, according to 9. In Subsection 5.1, the admissibility conditions in 9] are revisited, obtaining an extension of the results in that reference which include non-necessarily sequential topologies (Theorem 5.4). In Subsection 5.2, the following aim is achieved. Assume that the Hausdorff separability between points of $M$ and $\partial M$ is incorporated as one of these admissibility properties that characterize the c-boundary. Then the corresponding topology becomes equal to the chronological topology refined in the general $D$-separating way explained in Section 3 , under the hypothesis that $L_{c h r}$ is of first order and even under less restrictive hypothesis which exhibit the accuracy of the approach (see Theorem 5.8 the discussion in Remark 5.9 and the summarizing Figure 3).

The article finishes with an Appendix containing examples which show that most of the subtleties suggested by our procedures can occur effectively.

\section{Limit operators and sequential spaces}

In this section we develop some properties about limit operators and sequential spaces. There is a well established literature on the later, see [12, 16] and references therein for general background.

\subsection{General properties}

Let $X$ be an arbitrary set, let $S(X)$ the set of all sequences in $X$ and $\mathcal{P}(X)$ the set of parts of $X$. Following [9, Sect. 3.6], [11, Sect.5.2.2] we consider: 
Definition 2.1 A map $L: S(X) \rightarrow \mathcal{P}(X)$ is a limit operator if it satisfies the following compatibility condition for subsequences:

$$
L(\sigma) \subset L(\kappa), \quad \text { for any } \sigma, \kappa \in S(X) \text { with } \kappa \subset \sigma .
$$

In this case, the topology derived from $L$ is the topology $\tau_{L}$ whose closed sets are those subsets $C$ of $X$ such that $L(\sigma) \subset C$ for any sequence $\sigma \subset C$.

In fact, the compatibility condition for subsequences allows to prove easily that the so-defined closed subsets satisfy the axioms for a topology.

Remark 2.2 (1) We can assume with no loss of generality that any limit operator is coherent in the sense that, for any $x \in X$ one has $x \in L\left(\left\{x_{n}=x\right\}\right)$. This will be assumed explicitly in the case of limit operators of $k$-th order below.

(2) If a topology $\tau$ on $X$ has been prescribed, one can define a unique associated limit operator $L_{\tau}$ which gives directly the convergence of sequences in $(X, \tau)$ (i.e., such that the converse of formula (3) also holds), namely:

$$
L_{\tau}: S(X) \rightarrow \mathcal{P}(X), \quad L_{\tau}(\sigma):=\{p \in X: \sigma \rightarrow p \text { with } \tau\} .
$$

(3) For an arbitrary limit operator $L$, the derived topology satisfies clearly the following implication:

$$
p \in L(\sigma) \Longrightarrow \sigma \rightarrow p \text { with the topology } \tau_{L} .
$$

However, the converse does not hold in general and we will be also interested in this possibility. A trivial (non-coherent) example is just the limit operator $L(\sigma)=\emptyset$ for all $\sigma \in S(X)$, whose derived topology is the discrete one. A coherent example can be constructed as follows. Let $(X, \tau)$ be any sequential topological space with a convergent sequence $\sigma=\left\{x_{n}\right\} \rightarrow x_{\infty}$ such that no subsequence of $\sigma$ is equal to $\sigma$, except $\sigma$ itself (for example, the elements of $\sigma$ could be all distinct as in the case $\left.(X, \tau)=\mathbb{R}, \sigma=\{1 / n\}, x_{\infty}=0\right)$. Let $L_{\tau}$ be the associated limit operator and define a new limit operator $L$ such that $L(\tilde{\sigma})=\emptyset$, where $\tilde{\sigma}$ is any sequence that contains $\sigma$ as a subsequence, and $L(\mu)=L_{\tau}(\mu)$ for any other sequence $\mu$. As $\mu$ can be chosen equal to any subsequence of $\sigma$ (except $\sigma$ itself), this implies that, in any case, $\sigma$ will converge to $x_{\infty}$ with $\tau_{L^{\prime}}$ and, then, $\tau_{L^{\prime}}=\tau$.

Obviously, these examples correspond to limit operators where $L$ is regarded as empty on a sequence in a highly arbitrary way. Nevertheless, one may find naturally the following situation in the case of non-Hausdorff topologies: the limit operator yields a infinite set of limits, and these limits will yield naturally more limits. This will lead to the notion of $k$-th limit operator, to be studied below.

The considerations above motivate the following definition.

Definition 2.3 Let $D$ be a subset of $X$. We will say that a limit operator $L$ on $X$ is of first order on $D$ if, for any sequence $\sigma \subset D$ and any $p \in D$ :

$$
p \in L(\sigma) \Longleftrightarrow \sigma \rightarrow p \text { with } \tau_{L} .
$$

When $L$ is of first order on $D=X$ (i.e. $L=L_{\tau_{L}}$ ) then we say simply that $L$ is of first order.

As a first simple property to be used in the remainder, we have the following.

Lemma 2.4 Let $L, L^{\prime}$ be two limit operators on $X$ with derived topologies $\tau_{L}$ and $\tau_{L^{\prime}}$ resp. If

$$
L^{\prime}(\sigma) \subset L(\sigma) \quad \text { for any sequence } \sigma \subset X,
$$

then $\tau_{L} \subset \tau_{L^{\prime}}$. Moreover, when $L$ is of first order, the converse also holds. 
Proof. Let $C$ be a closed set for $\tau_{L}$, that is, $L(\sigma) \subset C$ for any sequence $\sigma \subset C$. As $L^{\prime}(\sigma) \subset$ $L(\sigma) \subset C$, we deduce that $C$ is also closed for $\tau_{L^{\prime}}$, and so, $\tau_{L} \subset \tau_{L^{\prime}}$.

For the converse, assume that $L$ is of first order and $q \in L^{\prime}(\sigma)$ for some sequence $\sigma \subset X$. Then, $\sigma \rightarrow q$ for $\tau_{L^{\prime}}$, and, by the inclusion $\tau_{L} \subset \tau_{L^{\prime}}$, this limit also holds for $\tau_{L}$. As $L$ is of first order, necessarily $q \in L(\sigma)$, as required.

Recall that a topological space $(X, \tau)$ is called sequential if any sequentially closed set (i.e. a set which contains all the limits of any sequence contained in it) is also a closed set. Note that the converse is true in any topological space.

Proposition 2.5 Let $X$ be a set:

(a) For any limit operator $L$ on $X$, its derived topology $\tau_{L}$ is sequential.

(b) For any topology $\tau$ on $X$, its associated limit operator $L_{\tau}$ (see formula (2)) has a derived topology $\tau_{S e q}:=\tau_{L_{\tau}}$, which is the coarsest one among the sequential topologies containing $\tau$. Moreover, $L_{\tau}$ is a limit operator of first order for $\tau_{S e q}$.

(c) A topology $\tau$ on $X$ is sequential if and only if $\tau=\tau_{\text {Seq. }}$.

Proof. (a) is straightforward from the definitions.

For (b), the inclusion $\tau \subset \tau_{S e q}$ follows from the fact that closed subsets are sequentially closed for any topology. Let $\tau^{\prime}$ be another sequential topology with $\tau \subset \tau^{\prime}$. If $\sigma$ converges to $p$ with $\tau^{\prime}$, then $\sigma \rightarrow p$ with $\tau$, which implies $p \in L_{\tau}(\sigma)$. Hence, sequentially closed sets for $\tau_{S e q}$ are also sequentially closed for $\tau^{\prime}$, and so, $\tau_{S e q} \subset \tau^{\prime}$. For the first order character of $L_{\tau}$, assume that $\sigma$ converges to $p$ with $\tau_{\text {Seq }}$. Since $\tau \subset \tau_{\text {Seq }}$, the sequence $\sigma$ must converge to $p$ with $\tau$, and thus, $p \in L_{\tau}(\sigma)$.

Finally, the right implication of (c) follows from the assumption that sequentially closed for $\tau$ implies closed (to the left, use (a)).

Remark 2.6 Summing up, a topological space $(X, \tau)$ is sequential if and only if $\tau=\tau_{L}$ for some limit operator $L$ on $X$, which can be chosen of first order (and, then, equal to $L_{\tau}$ ). From the previous properties and the known ones about sequential spaces (see [16], especially its figures 1.1 and 1.3) the following observations are in order:

(1) The map $\tau \mapsto L_{\tau}$ from the set $\mathcal{T}(X)$ of all the topologies on $X$ to the set of all the first order limit operators on $X$, is an onto map (as $L=L_{\tau_{L}}$ ). Nevertheless, it is not one to one, as any non-sequential topology $\tau$ has associated the limit operator $L_{\tau}$ equal to the limit operator for the sequential topology $\tau_{S e q}$. In fact, one has then an onto map

$$
\mathcal{T}(X) \rightarrow \mathcal{T}_{\text {Seq }}(X), \quad \tau \mapsto \tau_{\text {Seq }}
$$

where $\mathcal{T}_{\text {Seq }}(X)$ is the set of all the sequential topologies on $X$.

(2) Sequential spaces generalize Fréchet-Uryshon spaces (namely, the closure of a set consists of the limits of all the sequences in that set) and, then, first countable spaces, which include all the metrizable ones; however, sequential spaces can be regarded as quotients of metrizable spaces.

If $X$ is a sequential space, the continuity of a map $f: X \rightarrow Y$ into a topological space $Y$ can be characterized by the preservation of the limits of sequences. Nevertheless, the closure of a set $A \subset X$ may contain strictly the set of all the limits of sequences in $A$ (in contrast with FréchetUryshon), the uniqueness of the limits of sequences does not imply Hausdorffness (in contrast with first countability), and sequential compactness (i.e., the property that any sequence admits a convergent subsequence) plus Hausdorffness and first countability, do not imply compactness (in contrast with metrizability). Nevertheless, for sequential spaces, sequential compactness becomes equivalent to countable compactness (see for example [16, Proposition 3.2]) and, as the latter is weaker than compactness, compact sequential spaces are sequentially compact. 
The property of being sequential is not inherited by arbitrary subspaces, but it is inherited by open (or closed) subspaces [12, Prop. 1.9]. More sharply, the limit operators can be inherited by open subspaces, in the following sense.

Proposition 2.7 Let $D$ be an open subset of a sequential space $\left(X, \tau_{L}\right)$. The topology $\left.\tau_{L}\right|_{D}$ induced on $D$ by $\tau_{L}$ coincides with the topology $\tau_{\left.L\right|_{D}}$ derived from the restricted limit operator $\left.L\right|_{D}$ given by

$$
\left.L\right|_{D}(\sigma):=L(\sigma) \cap D, \quad \text { for any sequence } \sigma \subset D .
$$

Proof. It is straightforward to check that $\left.L\right|_{D}$ is a limit operator if so is $L$. So, we focus on the identification between $\left.\tau_{L}\right|_{D}$ and $\tau_{\left.L\right|_{D}}$.

In order to prove that $\left.\tau_{L}\right|_{D} \subset \tau_{\left.L\right|_{D}}$, let $C$ be a closed set of $\left.\tau_{L}\right|_{D}$. Then, $C=C^{\prime} \cap D$ with $C^{\prime}$ a closed set of $\tau_{L}$. Let $\sigma$ be any sequence in $C$. As $\sigma \subset C \subset C^{\prime}$ and $C^{\prime}$ is closed for $\tau_{L}$, necessarily $L(\sigma) \subset C^{\prime}$. Therefore,

$$
\left.L\right|_{D}(\sigma)=L(\sigma) \cap D \subset C^{\prime} \cap D=C \text {, and thus, } C \text { is closed for } \tau_{\left.L\right|_{D}} .
$$

For the inclusion $\left.\tau_{\left.L\right|_{D}} \subset \tau_{L}\right|_{D}$, let $B \subset D$ an open set of $\tau_{\left.L\right|_{D}}$. It is enough to prove that $B$ is also open for $\tau_{L}$. So, assume by contradiction that $X \backslash B$ is not closed for $\tau_{L}$, and thus, there exist a sequence $\sigma \subset X \backslash B$ and a point $p \in B \subset D$ such that $p \in L(\sigma)$. As $D$ is open for $\tau_{L}$, $\sigma \subset D$ eventually. In particular, $p \in L(\sigma) \cap D=\left.L\right|_{D}(\sigma)$, and from (3), $\sigma$ converges to $p$ with $\tau_{\left.L\right|_{D}}$. But $B$ is open for $\tau_{\left.L\right|_{D}}$, hence $\sigma$ eventually intersects $B$, which is a contradiction.

\subsection{Higher order limit operators}

When a limit operator $L$ is not of first order, $L(\sigma)$ does not provide all the topological limits of some sequence $\sigma \subset X$, that is, $L(\sigma) \varsubsetneqq L_{\tau_{L}}(\sigma)$. Nevertheless, in view of the discussion in Remark 2.2 (as well as Example 6.2 below), it is natural to wonder when any point in $L_{\tau_{L}}(\sigma) \backslash L(\sigma)(\neq \emptyset)$ can be obtained by iterating successively the limit operator $L$. In order to formalize this idea, we will assume that $L$ is a coherent limit operator (Remark 2.2 (1)) and consider the following transfinite definition:

$L^{1}(\sigma):=L(\sigma), \quad L^{i}(\sigma):=\left\{p \in X: p \in L\left(\left\{p_{n}\right\}\right), \quad\left\{p_{n}\right\} \subset \cup_{j<i} L^{j}(\sigma)\right\} \quad$ for any ordinal $i \geq 2$.

Note that if $p \in L^{k}(\sigma)$, then $p \in L\left(\left\{p_{n}=p\right\}_{n}\right)$ with $\left\{p_{n}\right\}_{n} \subset L^{k}(\sigma) \subset \cup_{j<i} L^{j}(\sigma)$ for any $i \geq k$, and thus, $p \in L^{i}(\sigma)$. Therefore, the following property holds:

$$
L^{k}(\sigma) \subset L^{i}(\sigma) \quad \text { for any sequence } \sigma \text { and any ordinals } k \leq i \text {. }
$$

Proposition 2.8 The inclusion $L^{i}(\sigma) \subset L_{\tau_{L}}(\sigma)$ holds for any $\sigma \subset X$ and any ordinal $i$.

Proof. One needs to show that if $p \in L^{i}(\sigma)$ then $\sigma$ converges to $p$ with the topology $\tau_{L}$, i.e., if $\sigma=\left\{p_{n}\right\}$ and $p \in U$ for some open set $U$ of $\tau_{L}$, there exists $n_{0}$ such that $p_{n} \in U$ for all $n \geq n_{0}$.

The proof will follow by transfinite induction. For $i=1$, the result is known from (2). So, assume that it is true for all $j<i$, and let us prove it for $i$. From the definition of $L^{i}(\sigma)$, there exists a sequence $\left\{q_{n}\right\} \subset \cup_{j<i} L^{j}(\sigma)$ such that $p \in L\left(\left\{q_{n}\right\}\right)$. From (2) the sequence $\left\{q_{n}\right\}$ converges to $p$ with the topology $\tau_{L}$, and so, there exists some $n_{0}^{\prime}$ such that $q_{n_{0}^{\prime}} \in U$. Since $q_{n_{0}^{\prime}} \in \cup_{j<i} L^{j}(\sigma)$, necessarily $q_{n_{0}^{\prime}} \in L^{j}(\sigma)$ for some $j<i$, and the hypothesis of induction ensures that $\sigma$ converges to $q_{n_{0}^{\prime}}$ with $\tau_{L}$. Recalling that $q_{n_{0}^{\prime}} \in U$, there exists $n_{0}$ such that $p_{n} \in U$ for all $n \geq n_{0}$. Hence, $\sigma$ converges to $p$ with $\tau_{L}$.

Motivated by the notion of first order limit operator, we now establish the following definition. 
Definition 2.9 A coherent limit operator $L$ is of $k$-th order for some ordinal $k$ if

$$
L_{\tau_{L}}(\sigma)=L^{k}(\sigma), \quad \text { but } \quad L_{\tau_{L}}(\sigma) \neq L^{j}(\sigma) \quad \text { for any } j<k .
$$

Recall that the coherent limit example constructed in Remark 2.2 (3) is not of $k$-th order for any ordinal. As a direct consequence of (6), Proposition 2.8 and this definition, we also deduce:

Corollary 2.10 If a coherent limit operator $L$ is of $k$-th order then $L^{k}(\sigma)=L^{i}(\sigma)$ for any $\sigma \subset X$ and any ordinal $i \geq k$.

\section{$3 \mathbf{T}_{2}$-separating boundaries in the general and sequential cases}

\subsection{Separating for an arbitrary topology}

In this section we consider the following problem. Let $(X, \tau)$ be a topological space, and let $D \subset X$ be an open subset with some good topological properties, namely, Hausdorff and locally compact. Typically, $D$ will be a Lorentzian manifold and $X$ some topological completion, so that $D$ is dense in $X$. However, we do not impose a priori the density, allowing $X \backslash D$ to be a bigger "crust". Now, assume that there is some pair of points $p \in D, q \in X \backslash D$ that are not Hausdorff separated, and we look for a "minimal refinement of the topology around $\partial D$ " in order to ensure that such pairs are $T_{2}$-separated. Formally, we are looking for a new topology $\tau^{*}$ which satisfies the following requirements.

Definition 3.1 Let $(X, \tau)$ be a topological space, and let $D \subset X$ be an open subset which is Hausdorff and locally compact. A topology $\tau^{*}$ is minimally $D$-separating if it satisfies:

(A $\left.A_{\text {Fin }}\right)$ Refinement of $\tau$ : the topology $\tau^{*}$ is finer than $\tau$, i.e. $\tau \subset \tau^{*}$.

(A $\left.A_{\text {Sep }}\right) T_{2}$-separability of points of $D$ and $X \backslash D:$ for any $p \in D, q \in X \backslash D$, there exist $U \in \tau$ and $V \in \tau^{*}$ such that $p \in U, q \in V$ and $U \cap V=\emptyset$.

( $A_{\text {Min }}$ ) Minimality: $\tau^{*}$ is a minimal topology among those satisfying $\left(A_{\text {Fin }}\right)$ and $\left(A_{\text {Sep }}\right)$, i.e. no other topology satisfying conditions $\left(A_{F i n}\right)$ and $\left(A_{S e p}\right)$ is strictly coarser than $\tau^{*}$.

Remark 3.2 (1) From property $\left(\mathrm{A}_{\mathrm{Fin}}\right)$, the subset $D$ will be also open for $\tau^{*}$.

(2) The condition of minimality $\left(\mathrm{A}_{\mathrm{Min}}\right)$ is essential in order to avoid trivial topologies. For example, if we considered as $\tau^{*}$ the one generated by $\tau$ and the crust $X \backslash D$ as a subbasis, then $\tau^{*}$ would satisfy $\left(\mathrm{A}_{\mathrm{Fin}}\right)$ and $\left(\mathrm{A}_{\mathrm{Sep}}\right)$, but at the cost of a separability which would forbid any point in the $\tau$-boundary $\partial D$ to be $\tau^{*}$-continuously reachable from $D$ (i.e., the $\tau^{*}$-boundary of $D$ would be the empty set); a similar drawback would happen if one considered the topology generated by $\tau$ and the sets $\{q\}$ for every point $q \in X \backslash D$ which is not $\mathrm{T}_{2}$-separated from some point in $D$.

(3) There exists a small asymmetry in the condition of $T_{2}$-separability $\left(\mathrm{A}_{\mathrm{Sep}}\right)$, as the separating neighborhood $U$ is not only required to belong to $\tau^{*}$ but also to $\tau$. Notice that only $D$ is assumed to be a topological subspace with additional nice properties (Hausdorfness, local compactness). This asymmetry in $\left(\mathrm{A}_{\mathrm{Sep}}\right)$, plus the minimality in $\left(\mathrm{A}_{\mathrm{Min}}\right)$, will ensure that the topology $\tau^{*}$ preserves most of the original properties of $(X, \tau)$. Among them, $\tau$ and $\tau^{*}$ will induce the same topology on $D$ (see Theorem 3.3 below). If the asymmetry were not imposed, $\left.\tau\right|_{D} \neq\left.\tau^{*}\right|_{D}$ may occur (Example6.3).

Now, we can state the following general result for topological spaces. 
Theorem 3.3 Let $(X, \tau)$ be a topological space and $D \subset X$ be a Hausdorff locally compact open subset of $X$. The topology $\tau^{*}$ generated by the subbasis

$$
\mathcal{S}=\tau \cup\{X \backslash K: K \text { is a compact subset of } D\}
$$

is the unique topology which satisfies the properties $\left(A_{F i n}\right),\left(A_{S e p}\right)$ and $\left(A_{M i n}\right)$ above, i.e., the unique minimally $D$-separating topology. Moreover, the restrictions of $\tau^{*}$ and $\tau$ on $D$ coincide.

Proof. Clearly, $\tau^{*}$ satisfies $\left(\mathrm{A}_{\mathrm{Fin}}\right)$. In order to prove $\left(\mathrm{A}_{\mathrm{Sep}}\right)$, consider $p \in D$ and $q \in X \backslash D$. Let $K$ be a compact neighborhood of $p$ in $D$ and $U$ its interior. From the definition of the subbasis $\mathcal{S}$ in (7), $V:=X \backslash K$ is open for $\tau^{*}$ and, so, $U$ and $V$ are the required open sets.

In order to prove $\left(\mathrm{A}_{\mathrm{Min}}\right)$ and the uniqueness, we will show that $\tau^{*} \subset \tau^{\prime}$ for any other topology $\tau^{\prime}$ satisfying $\left(\mathrm{A}_{\mathrm{Fin}}\right)$ and $\left(\mathrm{A}_{\mathrm{Sep}}\right)$. Since $\tau \subset \tau^{\prime}$, it suffices to show that $X \backslash K \in \tau^{\prime}$ for any compact subset $K$ of $D$. So, let us prove that $X \backslash K$ is a neighborhood of any $q \in X \backslash K$. From the property $\left(\mathrm{A}_{\mathrm{Sep}}\right)$ and the Hausdorffness of $D$, for every $p \in K$ there exists $U_{p} \in \tau \subset \tau^{\prime}$ and $V_{p} \in \tau^{\prime}$ such that $p \in U_{p}, q \in V_{p}$ and $U_{p} \cap V_{p}=\emptyset$. Since $K$ is compact, there exists $\left\{U_{p_{i}}\right\}_{i=1}^{n} \subset\left\{U_{p}\right\}_{p \in K}$ such that $K \subset \cup_{i=1}^{n} U_{p_{i}}$. Then, $V:=\cap_{i=1}^{n} V_{p_{i}} \in \tau^{\prime}$ satisfies $q \in V$ and $V \cap K \subset V \cap\left(\cup_{i=1}^{n} U_{p_{i}}\right)=\emptyset$. Therefore, $X \backslash K$ is a neighborhood of $q$, as required.

For the last assertion, it suffices to show that $A \cap D \in \tau$ for any $A \in \mathcal{S}$ (recall that $\mathcal{S}$ is subbasis of $\tau^{*}$ ). So, assume that $A=X \backslash K$, where $K$ is a compact subset of $D$ (if $A \in \tau$, the conclusion follows trivially from $D \in \tau)$. Since $D$ is Hausdorff, $K$ is closed in $D$, and thus, $D \backslash K(=A \cap D) \in \tau$, as required.

\subsection{Separating among sequential topologies}

Next, we consider the same problem as before but restricting our attention to sequential spaces. To do that, first we have to introduce the following adapted version of Definition 3.1:

Definition 3.4 Let $\left(X, \tau\left(=\tau_{L}\right)\right)$ be a sequential topological space, and let $D \subset X$ be an open subset which is Hausdorff and locally compact. A topology $\tau_{\text {Seg }}^{*}$ is sequentially minimally $D$ separating if it satisfies $\left(A_{F i n}\right)$ and $\left(A_{S e p}\right)$ from Definition 3.1. and the following minimality condition:

$\left(A_{M i n}^{\text {Seq }}\right)$ Sequential minimality: $\tau_{\text {Seq }}^{*}$ is a minimal topology among the sequential ones satisfying $\left(A_{\text {Fin }}\right)$ and $\left(A_{S e p}\right)$.

Now, we can state the following result for sequential topological spaces:

Theorem 3.5 Let $\left(X, \tau\left(=\tau_{L}\right)\right)$ be a sequential topological space, and let $D \subset X$ be a Hausdorff locally compact open subset of $X$. The topology $\left(\tau^{*}\right)_{\text {Seq }}$ (where $\tau^{*}$ is the unique minimally $D$ separating topology according to Theorem 3.3 and the notation introduced in Proposition 2.5(b) is used), is the unique sequentially minimally $D$-separating topology, that is. $\tau_{\text {Seq }}^{*}$ exists, it satisfies $\left(A_{\text {Min }}^{\text {Seq }}\right)$ as an unique minimum and $\left(\tau^{*}\right)_{\text {Seq }}=\tau_{\text {Seq }}^{*}$. Moreover, the restrictions of $\tau_{\text {Seq }}^{*}$ and $\tau$ coincide on $D$.

Proof. From Proposition 2.5 (b), the topology $\left(\tau^{*}\right)_{\text {Seq }}$ is the coarsest topology among the sequential ones containing $\tau^{*}$. So, $\left(\tau^{*}\right)_{\text {Seq }}$ clearly satisfies conditions $\left(\mathrm{A}_{\mathrm{Fin}}\right)$ and $\left(\mathrm{A}_{\mathrm{Sep}}\right)$, as these conditions are already satisfied by $\tau^{*}$. Moreover, any other sequential topology $\tau^{\prime}$ satisfying $\left(\mathrm{A}_{\mathrm{Fin}}\right)$ and $\left(\mathrm{A}_{\mathrm{Sep}}\right)$, must contain $\tau^{*}$. So, from the coarsest character of $\left(\tau^{*}\right)_{\text {Seq }}$, the topology $\tau^{\prime}$ must contain $\left(\tau^{*}\right)_{S e q}$. Therefore, we deduce both, $\left(\tau^{*}\right)_{S e q}$ satisfies condition $\left(\mathrm{A}_{\text {Min }}^{\text {Seq }}\right)$ and it is unique. 
The last assertion follows from Proposition 2.7 and the fact that, for all $p \in D$ and all sequence $\sigma \subset D$, the following chain of equivalences holds

$$
p \in L_{\tau}(\sigma) \Longleftrightarrow \sigma \text { converges to } p \text { with } \tau \Longleftrightarrow \sigma \text { converges to } p \text { with } \tau^{*} \Longleftrightarrow p \in L_{\tau^{*}}(\sigma),
$$

where the first equivalence follows as $L_{\tau}$ is the (first order) limit operator associated to $\tau$, the second one from the last assertion of Theorem 3.3 and the third one from the definition of $L_{\tau^{*}}$.

This result provides an elegant solution to our problem. However, one can wonder for a better understanding of $\tau_{\text {Seq }}^{*}$. Notice that the topology $\tau_{S e q}^{*}$ is defined in terms of the limit operator $L_{\tau^{*}}$, which is not directly based on some limit operator $L$ of $\tau$, but on the topology $\tau^{*}$. This is a difficulty in order to manage such a topology for practical purposes.

\subsection{Limit operators and further properties of the separating topology}

This subsection is devoted to find an alternative limit operator $L^{*}$, directly constructed from $L$, whose derived topology $\tau_{L^{*}}$ coincides with the sequentially minimally $D$-separating one $\tau_{\text {Seq }}^{*}$. The natural candidate is:

$$
L^{*}(\sigma):=\left\{\begin{array}{lc}
L(\sigma) \cap D & \text { if } \exists \kappa \subset \sigma \text { and } p \in D \text { such that } p \in L(\kappa) \\
L(\sigma) & \text { otherwise }
\end{array}\right.
$$

(so, $L^{*}(\sigma)=L(\sigma) \cap D$ not only when $L(\sigma) \cap D \neq \emptyset$ but also when $L(\kappa) \cap D \neq \emptyset$ for some subsequence $\kappa$; this is necessary in order to ensure that $L^{*}$ is a limit operator, see Proposition 3.7). Indeed, the definition of $L^{*}$ suggests that it is the smallest modification of $L$ such that no sequence $\sigma \subset X$ will converge to both, $p \in D$ and $q \in X \backslash D$. However, a caution must be taken into account: this property will be ensured only if $L^{*}$ is of first order - otherwise, $\sigma$ might

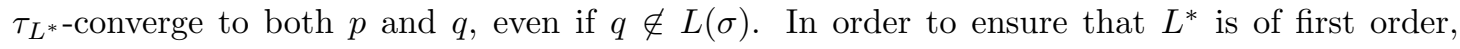
a natural requirement will be to assume that so is $L$. Of course, this is not restrictive from a fundamental viewpoint, as one can always replace it by the associated limit operator $L_{\tau}$ in (2), which is of first order. However, this caution must be taken into account from a practical viewpoint, when $L^{*}$ is being computed from a sequential topology $\tau$ constructed by means of some prescribed limit operator $L$ (as in the case of some commented relativistic boundaries).

With this aim for $L^{*}$, notice first that formula (4) in Lemma 2.4 can be regarded as a characterization of the property $\left(\mathrm{A}_{\mathrm{Fin}}\right)$ of Defn. 3.1 in terms of the limit operator $L$. It is convenient to rewrite also the condition $\left(\mathrm{A}_{\mathrm{Sep}}\right)$ in such terms as follows.

Lemma 3.6 Let $L, L^{\prime}$ be two limit operators on $X$ with derived topologies $\tau_{L}$ and $\tau_{L^{\prime}}$ resp., and let $D$ be open, locally compact and Hausdorff for $\tau_{L}$. Assume also that $\tau_{L} \subset \tau_{L^{\prime}}$.

If the topology $\tau_{L^{\prime}}$ satisfies the condition $\left(A_{S e p}\right)$ (with $\tau^{*} \equiv \tau_{L^{\prime}}, \tau \equiv \tau_{L}$ ), then one has:

$$
\text { when } L^{\prime}(\sigma) \cap D \neq \emptyset \text { for some } \sigma \subset X \text {, then } L^{\prime}(\sigma) \subset D \text {. }
$$

The converse is true if we assume that $L$ is of first order on $D$ and, additionally:

$$
\left.L\right|_{D}(\sigma)=\left.L^{\prime}\right|_{D}(\sigma) \text { for any sequence } \sigma \subset D
$$

(so that $\left.\tau_{L}\right|_{D}=\left.\tau_{L^{\prime}}\right|_{D}$ by Proposition 2.7).

Proof. To the right, observe that if (9) does not hold, then there exist $p \in D, q \in X \backslash D$ and a sequence $\sigma \subset X$ such that $p, q \in L^{\prime}(\sigma)$. Then, from (3), $\sigma$ converges with $\tau_{L^{\prime}}$ to both $p, q$, which contradicts that $\tau_{L^{\prime}}$ satisfies $\left(\mathrm{A}_{\mathrm{Sep}}\right)$. 
For the converse, for each $p \in D$, take some compact (for $\tau_{L}$ of, equally by 10 , for $\tau_{L^{\prime}}$ ) neighborhood $K \subset D$ of $p$, and let $U \in \tau_{L}$ be its $\tau_{L}$-interior. The property ( $\mathrm{A}_{\mathrm{Sep}}$ ) will follow trivially if we prove that $X \backslash K$ is open for $\tau_{L^{\prime}}$, that is, if $L^{\prime}(\sigma) \subset K$ for any sequence $\sigma \subset K$. So, assume by contradiction that $q \in L^{\prime}(\sigma) \backslash K$ for some sequence $\sigma \subset K$. Then $\sigma \rightarrow q$ with $\tau_{L^{\prime}}$ and, therefore, with $\tau_{L}$. Note that $K$ is a compact subset of $D$ which contains $\sigma$, and both, $D$ and $K$ are sequential spaces with the restriction of $\tau_{L}$ (use either [12, Prop. 1.9] or Proposition 2.7 for the sequentiality of $D$ and, then, [16, Lemma 3.7] for $K$ ). So, the compactness of $K$ implies its sequential compactness (see the end of Remark 2.6) and there exists a subsequence $\kappa \subset \sigma$ and some $p^{\prime} \in K \subset D$ such that $\kappa \rightarrow p^{\prime}$ with $\tau_{L}$. Since $L$ is of first order on $D$, necessarily $p^{\prime} \in L(\kappa)$ which, combined with 10$)$, implies $p^{\prime} \in L^{\prime}(\kappa)$. On the other hand, by hypothesis, $q \in L^{\prime}(\sigma) \backslash K$. So, there are two possibilities: either $q \in D$, which contradicts the Hausdorffness of $\left.\tau_{L^{\prime}}\right|_{D}=\left.\tau_{L}\right|_{D}$, or $q \in X \backslash D$, which contradicts property (9) applied to $\kappa$.

The hypotheses under which the condition $(9)$ characterizes $\left(A_{\text {Sep }}\right)$ are technical, but they will become natural in the applications of Lemma 3.6. Next, let us characterize $L^{*}$ for any limit operator $L$.

Proposition 3.7 Let $\left(X, \tau_{L}\right)$ be a sequential topological space and $D \subset X$ a Hausdorff locally compact open subset of $X$. Then, $L^{*}$ is a limit operator which satisfies the properties (4), (9) and (10), and it is the maximum operator satisfying them, that is: if $L^{\prime}$ is another limit operator satisfying (4), (9) and (10), then $L^{\prime} \subset L^{*}$ (i.e., $L^{\prime}(\sigma) \subset L^{*}(\sigma)$ for any sequence $\left.\sigma \subset X\right)$.

Proof. First, let us prove that $L^{*}$ is a limit operator, i.e. it satisfies (1). Assume by contradiction that there exist a sequence $\sigma$ and a subsequence $\kappa$ such that $L^{*}(\sigma) \not \subset L^{*}(\kappa)$. Recall that $L^{*}(\sigma) \subset L(\sigma) \subset L(\kappa)$. Hence, necessarily $L^{*}(\kappa) \neq L(\kappa)$. In particular, $L(\kappa) \cap D \neq \emptyset$, and thus, $L^{*}(\sigma)=L(\sigma) \cap D \subset L(\kappa) \cap D=L^{*}(\kappa)$, in contradiction with the initial hypothesis.

From the definition of $L^{*}$, it satisfies the properties (4), (9) and (10). So, it remains to prove the maximal character of $L^{*}$. Let $L^{\prime}$ be another limit operator satisfying such properties. In order to prove that $L^{\prime}(\sigma) \subset L^{*}(\sigma)$ for any sequence $\sigma \subset D$, consider the cases:

- There exists $\kappa \subset \sigma$ and $p \in D$ such that $p \in L(\kappa)$. Observe that, as $D$ is open, we can assume $\kappa \subset D$. Then, $L^{*}(\sigma)=L(\sigma) \cap D$ from the definition (8) and $p \in L^{\prime}(\kappa)$ as $L^{\prime}$ satisfies 10 . Assume by contradiction that $L^{\prime}(\sigma) \not \subset L^{*}(\sigma)$. There exists $q \in L^{\prime}(\sigma)$ such that $q \notin L^{*}(\sigma)$ and, necessarily $q \notin D$ by 10 . So, $q$ belongs to both $L^{\prime}(\kappa)$ and $X \backslash D$, which absurd as $p \in L^{\prime}(\kappa) \cap D$ and $L^{\prime}$ satisfies $(9)$.

- There are no $\kappa \subset \sigma$ under previous conditions. Then, $L^{*}(\sigma)=L(\sigma)$, and, from (4), one has $L^{\prime}(\sigma) \subset L(\sigma)=L^{*}(\sigma)$.

The following technical assertion will be useful; notice that Lemma 3.6 gives conditions for its applicability.

Lemma 3.8 Assume that $\tau_{L^{*}}$ satisfies $\left(A_{S e p}\right)$. If $\sigma \rightarrow q$ with $\tau_{L^{*}}$ and $q \in L(\sigma)$, then $q \in L^{*}(\sigma)$.

Proof. If $q \in D$ then $q \in L(\sigma) \cap D=L^{*}(\sigma)$, as required. So, assume that $q \in X \backslash D$. It suffices to prove that $L(\kappa) \cap D=\emptyset$ for any subsequence $\kappa \subset \sigma$ (since then $q \in L(\sigma)=L^{*}(\sigma)$ ). Assuming by contradiction that there exists $p \in L(\kappa) \cap D \neq \emptyset$, the sequence $\kappa$ converges to both, $p$ and $q$, with $\tau_{L^{*}}$, in contradiction with property $\left(\mathrm{A}_{\mathrm{Sep}}\right)$.

Now, we can establish the following result that clarifies the role of limit operators:

Theorem 3.9 Let $\left(X, \tau \equiv \tau_{L}\right)$ be a sequential topological space and let $D \subset X$ be a Hausdorff locally compact open subset of $X$. Assume that the limit operator $L$ is of first order on $D$. Then, the following statements hold: 
(i) The topology derived by the limit operator $L^{*}$ defined on $\sqrt{8}$ ) is finer than the unique sequentially minimally D-separating topology. So, we have the following chain of topologies:

$$
\tau \subset \tau^{*} \subset \tau_{S e q}^{*} \subset \tau_{L^{*}}
$$

(ii) If $L$ is of first order (on all $X$ ), then $L^{*}$ is also of first order and both topologies $\tau_{\text {Seq }}^{*}$ and $\tau_{L^{*}}$ coincide.

Proof. For (i), let us consider a set $C$ which is closed set for the topology $\tau_{\text {Seq }}^{*}$ and prove that such a set is also closed for $\tau_{L^{*}}$. Our aim is to show that $L^{*}(\sigma) \subset C$ for all sequence $\sigma \subset C$. Consider a point $p \in L^{*}(\sigma)$ and recall that, from the definition of $L^{*}$, we may consider two cases. For the first one, assume that $L^{*}(\kappa) \cap D \neq \emptyset$ for some subsequence $\kappa \subset \sigma$, and so, that $p \in L^{*}(\sigma)=L(\sigma) \cap D$. Then, $\sigma$ converges to $p$ with $\tau$ and $\sigma$ will abandon any compact set of $D$ not containing the point $p$ (recall that $D$ is Hausdorff). Therefore, $\sigma$ converges to $p$ with $\tau^{*}$ and, from the definition of its limit operator, $p \in L_{\tau^{*}}(\sigma)$. But $\tau_{S e q}^{*}=\left(\tau^{*}\right)_{S e q}$ (Theorem 3.5p and $C$ is closed for $\tau_{\text {Seq }}^{*}$, so $p \in C$.

For the second case, assume that $L(\kappa) \cap D=\emptyset$ for all subsequence $\kappa \subset \sigma$, and so, $p \in X \backslash D$. As $p \in L^{*}(\sigma)$, we also have that $p \in L(\sigma)$, and so, $\sigma$ converges to $p$ with $\tau$. Moreover, such a sequence will converge to $p$ also with $\tau^{*}$ : otherwise, there would be a compact $K$ and a subsequence $\kappa \subset K$ of $\sigma$ converging to a point $q \in D$ with the topology $\tau$. However, as $L$ is of first order on $D$, this would imply that $q \in L(\kappa) \cap D \neq \emptyset$, a contradiction. So, $\sigma$ converges to $p$ with $\tau^{*}$ and, thus, $p \in L_{\tau^{*}}(\sigma)$. In conclusion, as $C$ is closed for $\tau_{\text {Seq }}^{*}$, again $p \in C$, as desired. To finish (i), recall that the two first inclusions in (11) are obvious from $\left(\mathrm{A}_{\mathrm{Fin}}\right)$ in Defn. 3.1 and Theorem 3.5 (notice also Prop. 2.5 (b)), respectively.

Now, let us prove (ii). For the first order character of $L^{*}$, observe first that since $L^{*}$ satisfies properties (4), (9) and (10) (recall Proposition 3.7), and $L$ is of first order, Lemmas 2.4, 3.6. ensure that $\tau_{L^{*}}$ satisfies $\left(\mathrm{A}_{\text {Fin }}\right)$ and $\left(\mathrm{A}_{\mathrm{Sep}}\right)$. Now assume that $\sigma \rightarrow q$ with $\tau_{L^{*}}$ for some sequence $\sigma \subset X$. From $\left(\mathrm{A}_{\text {Fin }}\right), \sigma \rightarrow q$ with $\tau_{L}$ and, as $L$ is of first order, $q \in L(\sigma)$. Then, Lemma 3.8 applies, and $q \in L^{*}(\sigma)$ follows, as desired.

For the second assertion of (ii), recall that from (i), we have the inclusion $\tau_{S e q}^{*} \subset \tau_{L^{*}}$. As $L_{\tau^{*}}$ is a first order limit operator, Lemma 2.4 ensures that $L^{*}(\sigma) \subset L_{\tau^{*}}(\sigma)$ for all sequence $\sigma \subset X$. So, taking into account Proposition 3.7, it suffices to prove that $L_{\tau^{*}}$ satisfies properties (4), (9) and (10). Properties (4) and $(9)$ follow from properties $\left(A_{\text {Fin }}\right)$ and $\left(A_{S e p}\right)$ (recall Lemmas 2.4. 3.6) while (10) is a consequence of Theorem 3.5 .

Remark 3.10 As announced at the beginning of the present subsection, the hypotheses of being $L$ of first order is necessary in Theorem 3.9 (even though it is not in Proposition 3.7), but this is not a fundamental restriction because, given a sequential topology $\tau$, one can consider its (first order) limit operator $L_{\tau}$ given in (2).

Nevertheless, Theorem 3.9 allows to understand better the role of $L$ and $L^{*}$ in order to obtain the separating topologies. Summing up, associated to a sequential topology $\tau$ writtten as $\tau=\tau_{L}$ we have defined three different topologies: $\tau^{*}, \tau_{\text {Seq }}^{*}\left(=\left(\tau^{*}\right)_{S e q}\right)$ and $\tau_{L^{*}}$. They always satisfy

$$
\tau \subset \tau^{*} \subset \tau_{S e q}^{*}
$$

and, whenever $\tau_{L^{*}}$ satisfies $\left(\mathrm{A}_{\text {Sep }}\right.$ ) (in particular, when $L$ is of first order on $\left.D\right), \tau_{\text {Seq }}^{*} \subset \tau_{L^{*}}$. The first constructed topology $\tau^{*}$ satisfies the required separating properties, but it is not necessarily sequential. The second $\tau_{\text {Seq }}^{*}$ satisfies both, the separating properties and sequentiality, even though it has a drawback from the practical viewpoint, namely, one does not have a priori an explicit expression for $L_{\tau^{*}}$ in terms of $L$. Finally, the third $\tau_{L^{*}}$ may depend on the choice of $L$ for the topology $\tau$, but it coincides with $\tau_{S e q}^{*}$ when $L$ is of first order (i.e. $L=L_{\tau}$ ). So, one obtains the explicit limit operator $L_{\tau^{*}}=L^{*}$. 


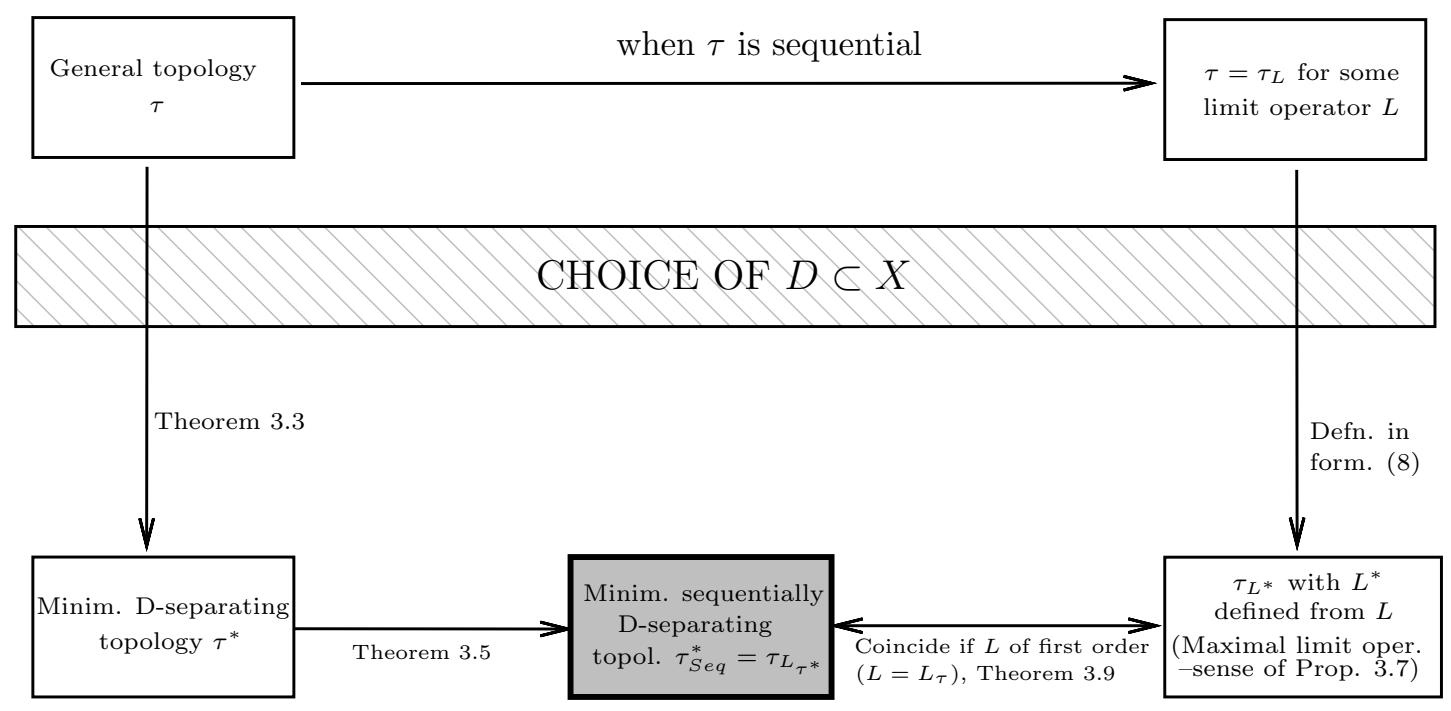

Figure 1: Summary of the constructions and results of Section 3 .

Although the first order restriction for $L$ is not fundamental in this context (see the previous remark), we will consider also non-necessarily first order limit operator $L$, as this might be the case for the natural operator for the chronological topology. So, the next proposition gives a small extension of the previous theorem, to be used later.

In what follows, $\tau$ will be a sequential topology derived from some limit operator $L$, i.e. $\tau=\tau_{L}$.

Lemma 3.11 If $p \in X \backslash D$ satisfies $p \in L_{\tau}^{*}(\sigma)$ for some sequence $\sigma \subset X$, then $L^{i}(\sigma)=\left(L^{*}\right)^{i}(\sigma)$ for any ordinal $i$.

Proof: The inclusion to the left is trivial, so we will focus on the inclusion to the right. We will proceed by transfinite induction. For $i=1$, the equality follows from the definition of $L^{*}$ and the fact that no subsequence $\kappa$ of $\sigma$ satisfies $d \in L(\kappa) \cap D$; in fact, otherwise, $d \in L_{\tau}(\kappa)$, and thus, $L_{\tau}^{*}(\sigma) \subset D$, which implies $p \notin L_{\tau}^{*}(\sigma)$, a contradiction. So, let us assume that the result is valid for any $j<i$, and let us prove it for $i$. Recall first that $L(\kappa) \cap D=\emptyset$ for any subsequence $\kappa$ of $\left\{p_{n}\right\} \subset \cup_{j<i} L^{j}(\sigma)$. In fact, otherwise, there exists some $d \in L(\kappa) \cap D$ for some subsequence $\kappa$ as before, and thus $d \in L^{i}(\sigma) \subset L_{\tau}(\sigma)$ (recall Proposition 2.8), which implies $L_{\tau}^{*}(\sigma) \subset D$, in contradiction to the fact that $p \in L_{\tau}^{*}(\sigma)$. So, consider a point $q \in L^{i}(\sigma)$. Then, there exists a sequence $\left\{p_{n}\right\} \subset \cup_{j<i} L^{j}(\sigma)=\cup_{j<i}\left(L^{*}\right)^{j}(\sigma)$ (recall the hypothesis of induction) such that $q \in$ $L\left(\left\{p_{n}\right\}\right)$. Since $L(\kappa) \cap D=\emptyset$ for any subsequence $\kappa$ of $\left\{p_{n}\right\}$, necessarily $q \in L\left(\left\{p_{n}\right\}\right)=L^{*}\left(\left\{p_{n}\right\}\right)$, and thus, $q \in\left(L^{*}\right)^{i}(\sigma)$.

Proposition 3.12 Let $L$ be a limit operator of $k$-th order (for some ordinal $k$ ) which is also of first order on D. If $L^{*}$ is of first order then $L^{*}=L_{\tau}^{*}\left(\right.$ where $\left.L_{\tau}^{*}:=\left(L_{\tau}\right)^{*}\right)$.

Proof: Since $L(\sigma) \subset L_{\tau}(\sigma)$ for all $\sigma \subset X$, and $L$ is of first order on $D$, we directly have

$$
L^{*}(\sigma) \subset L_{\tau}^{*}(\sigma) .
$$

For the inclusion to the left, consider a point $p \in L_{\tau}^{*}(\sigma)$. If $p \in D$ then

$$
p \in L_{\tau}^{*}(\sigma) \Rightarrow p \in L_{\tau}(\sigma) \Rightarrow p \in L(\sigma) \Rightarrow p \in L^{*}(\sigma),
$$


as required (we have used (8) for the first and third implications, and the first order character of $L$ on the open set $D$ for the second implication). If, otherwise $p \in X \backslash D$, from Lemma 3.11 . $L^{i}(\sigma)=\left(L^{*}\right)^{i}(\sigma)$ for any ordinal $i$. Moreover, as $L^{*}$ is of first order, $L^{*}(\sigma)=\left(L^{*}\right)^{i}(\sigma)$ for any ordinal $i$ (recall Corollary 2.10). Therefore, taking into account that $L$ is a limit operator of $k$-th order, we have

$$
p \in L_{\tau}^{*}(\sigma) \subset L_{\tau}(\sigma)=L^{k}(\sigma)=\left(L^{*}\right)^{k}(\sigma)=L^{*}(\sigma) .
$$

In conclusion, in both cases we deduce that $p \in L^{*}(\sigma)$, as desired.

\section{Completions of spacetimes, $T_{2}$-separability and c-boundary}

\subsection{A revision of some boundaries in Relativity}

Let $(M, g)$ be a spacetime, i.e. a connected time-oriented Lorentzian manifold. There are several types of boundaries in Relativity applicable to (some classes of) spacetimes, yielding a completion of the spacetime $\bar{M}=M \cup \partial M$. Among them, one has Geroch geodesic boundary (g-boundary) [13. Schmidt bundle boundary (b-boundary) [25, 26], Scott and Szekeres abstract boundary (aboundary) [24, Penrose conformal boundary [21, 28] and Geroch, Kronheimer and Penrose causal boundary (c-boundary).

The g-boundary was defined by using classes of incomplete geodesics in $(M, g)$. For the bboundary, one defines a certain positive definite metric on the bundle of linear frames $L M$ of $M$, takes the Cauchy completion of $L M$ and induces then a boundary for $(M, g)$. Both constructions satisfy the following a priori desirable properties pointed out by Geroch, Liang and Wald [15]:

(i) every incomplete geodesic in the original spacetime terminates at a point, and

(ii) they are geodesically continuous, in a sense rigorously defined in [15].

However, Geroch. et al. found a drawback for any boundary satisfying (i) and (ii): in a simple insightful example of (stably causal) spacetime, these two conditions implied the existence of a point $r$ in the spacetime and a point $s$ in the boundary non- $T_{1}$-separated (see Fig. 2); the authors proposed even a refined version of the example that was flat. The topology in the examples was always sequential and, moreover, there was a natural intuitive sense of convergence in the formulation of (ii). So, this drawback was regarded as a reason to reject a priori the topologies satisfying them.

Now, recall that our previous results can be applied. More precisely, putting $X$ equal to the union of $M$ and its g- or b- (or other) boundary $\partial M$, and $D=M$, one can modify slightly the original topology $\tau$ by taking the minimally $D$-separating one $\tau^{*}$ provided by Theorem 3.3 (or the sequentially minimally $D$-separating one $\tau_{\text {Seq }}^{*}$ in Theorem 3.5 if sequentially is also required to be preserved). This topology does not present the commented drawback and can be developed further. Of course, this modification of the topology will not solve magically all the problems of these boundaries, but it suggests possible improvements and opens the opportunity to re-study them (see, for instance, [23]).

Let us explain this briefly for the g-boundary (even though the b-boundary is more elegant and appealing mathematically 2, 17, it has other type of drawbacks from the physical viewpoint, see [5, 22]). As a previous question, one has to ensure that, if the spacetime is dense for the topology $\tau$ of the original completion, it is also dense for the modified one $\tau^{*}$. For this purpose, the following straightforward proposition is clearly applicable to the g-boundary, as well as to the b-, c- and conformal boundaries:

Proposition 4.1 Let $(X, \tau)$ be a topological space and $D \subset X$ a Hausdorff locally compact open subset of $X$. Assume that for any $x \in X \backslash D$ there exists some sequence $\sigma \subset D$ such that 
$x \in L_{\tau}(\sigma)$. Then, $D$ is dense in $X$ for $\tau$. If, in addition, such a $\sigma$ can be chosen such that it satisfies $L_{\tau}(\sigma)=L_{\tau}^{*}(\sigma)$, then $D$ is also dense for $\tau_{\text {Seq }}^{*}$.

Now, our modified topology $\tau^{*}$ for the g-boundary must violate one of the two properties (i) or (ii) above. Clearly, (i) will not be violated because of the minimality of our modification (recall the previous proposition). So, the key is the meaning of the hypothesis geodesically continuous in (ii). Geroch, Liang and Wald introduced a natural definition of this concept in terms of the exponential map. In addition, they gave a clever counterexample $(M, g)$ to the property of $T_{1}$ separability, by exploiting the following property (see Figure 2 as well as [15]): removing a point $s$ in Lorentz-Minkowski $\mathbb{L}^{2}$, they found a metric $g$ (conformal to the usual one) and two points $p, r \in M$ joined by a sequence of (future-directed) timelike geodesics $\gamma_{i}$ of length equal to 1 such that; (a) their initial velocities $\xi_{i}=\gamma_{i}^{\prime}(0)$ converge to a timelike vector $\xi$, and (b) the geodesic $\gamma$ with $\xi=\gamma^{\prime}(0)$ satisfy that $\lim _{t \nearrow_{1}} \gamma(t)$ is the removed point $s$. Their conclusion was that $s$ should be identified with a point of the g-boundary, and any neighborhood of this point contains $r$. Our modified topology $\tau^{*}$ separates $r$ and $s$, and seems to give a reasonble behavior for the topology in this particular example. Nevertheless, this does not simply mean that $\tau^{*}$ must be the right topology for the g-construction (notice that Geroch had also suggested in his original article the possibility of a modification of the g-topology, see the footnotes 10 and 14 in [13]). A closer look to Geroch et al.'s counterexample shows that they took advantage of the lack of compactness of $J^{+}(p) \cap J^{-}(r)$ as well as the lack of good convergence properties in the closure of a convex set. So, a first test for a redefinition might be carried out by restricting the class of spacetimes (for example, starting at globally hyperbolic ones). We leave this question as open for possible future studies.

The a-boundary, conformal boundary and c-boundary are not affected a priori by previous objection, as they are not formulated in terms of geodesics. The a-boundary 24 is defined in terms of open embeddings and has been developed systematically at the level of the set of ideal points. At the topological level, Barry and Scott have introduced recently two topologies for the a-boundary, the so-called attached and strongly attached point topologies (see [3, 4 for details). By construction, these topologies are not affected by the problem of lack of Hausdorffness between manifold and boundary points, and they have other desirable properties (in fact, their second proposal improves some properties of the first one). The conformal boundary is defined in terms of open conformal embeddings in a bigger (and Hausdorff) spacetime; so, it is not affected by these problems of separability. Nevertheless, harder problems appear in order to ensure the existence of such conformal embeddings and, in this case, the uniqueness of the so-obtained conformal boundary. The c-boundary appears as a natural alternative to the conformal boundary. In fact, the c-completion of spacetimes is a conformally invariant and systematic construction, applicable to any strongly causal spacetime.

\subsection{Basics on the c-boundary}

The c-completion of a strongly causal spacetime is constructed by adding ideal points to the spacetime in such a way that any timelike curve in the original spacetime acquires some endpoint in the new space. In the original article by Geroch, Kronheimer and Penrose [14], some doubts on its topology were pointed out. In fact, the original definition has suffered quite a few modifications. We will consider here the recent redefinition in [9] (which includes an approach to the topology of partial boundaries by Harris [18, 19]), and refer also there for extensive bibliography on the topic (see also [20, 10, 11] and references therein). This c-completion of a spacetime is always $T_{1}$, and there are situations where the possible non- $T_{2}$ separation of two points of the boundary appears as natural. However, there exist still examples where a point of $M$ and one 


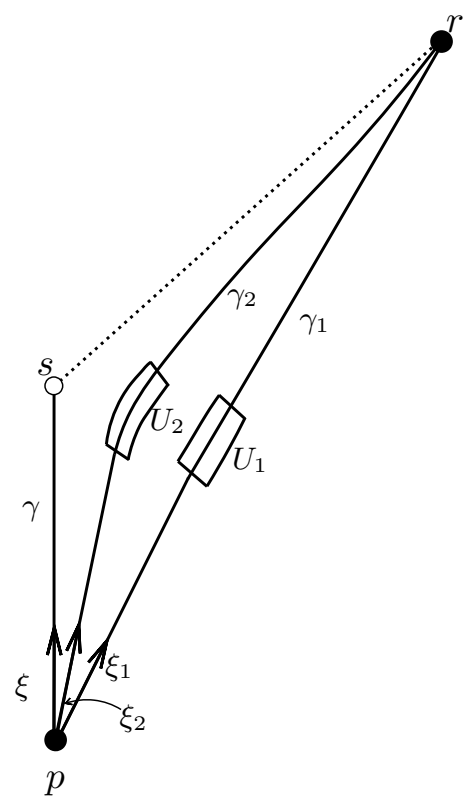

Figure 2: In their example, Geroch, Liang and Wald considered $\mathbb{R}^{2} \backslash\{s\}$ endowed with a metric $\Omega \cdot \eta$, where $\eta$ is the Minkowski metric and $\Omega$ is a conformal factor. The conformal factor was defined in such a way that all $\gamma_{i}$ are geodesics and $\Omega \equiv 1$ outside of the open sets $U_{i}$ as well as on each curve $\gamma_{i}$ inside $U_{i}$ (see [15] for details). 
of its c-boundary $\partial M$ are not $T_{2}$-related (see Example 6.1). As in the case of the g- and bboundaries, one can get rid of this inconvenience by using the minimal modification of the original topology in Theorems 3.3 and 3.5. In the remainder of Section 4, we will develop in detail this modification, in order to check that all the other desirable properties of the c-boundary are preserved by this minimal modification.

First, we will introduce some basic notions. A non-empty subset $P \subset M$ is called a past set if it coincides with its past; i.e., $P=I^{-}(P):=\{p \in M: p \ll q$ for some $q \in P\}$. The common past of $S \subset M$ is defined by $\downarrow S:=I^{-}(\{p \in M: \quad p \ll q \forall q \in S\})$. In particular, the past and common past sets are open. A past set that cannot be written as the union of two proper past sets is called indecomposable past set, IP. An IP which does coincide with the past of some point of the spacetime $P=I^{-}(p), p \in M$ is called proper indecomposable past set, PIP. Otherwise, $P=I^{-}(\gamma)$ for some inextendible future-directed timelike curve $\gamma$, and it is called terminal indecomposable past set, TIP. The dual notions of future set, common future, IF, PIF and TIF, are defined just by interchanging the roles of past and future in previous definitions.

To construct the future and past c-completion, first we have to identify each event (point) $p \in M$ with its $\mathrm{PIP}, I^{-}(p)$, and $\mathrm{PIF}, I^{+}(p)$. This is possible in any distinguishing spacetime, that is, a spacetime which satisfies that two distinct events $p, q$ have distinct chronological futures and pasts $\left(p \neq q \Rightarrow I^{ \pm}(p) \neq I^{ \pm}(q)\right)$. In order to obtain consistent topologies in the c-completions, we will focus on a somewhat more restrictive class of spacetimes, the strongly causal ones. These are characterized by the fact that the PIPs and PIFs constitute a sub-basis for the topology of the manifold $M$.

Now, every event $p \in M$ can be identified with its PIP, $I^{-}(p)$. So, the future c-boundary $\hat{\partial} M$ of $M$ is defined as the set of all the TIPs in $M$, and the future c-completion $\hat{M}$ becomes the set of all the IPs:

$$
M \equiv \mathrm{PIPs}, \quad \hat{\partial} M \equiv \mathrm{TIPs}, \quad \hat{M} \equiv \mathrm{IPs}_{\mathrm{s}}
$$

Analogously, each $p \in M$ can be identified with its PIF, $I^{+}(p)$. The past c-boundary $\partial{ } M$ of $M$ is defined as the set of all the TIFs in $M$, and the past $c$-completion $\check{M}$ is the set of all the IFs:

$$
M \equiv \mathrm{PIFs}, \quad \check{\partial} M \equiv \mathrm{TIFs}, \quad \check{M} \equiv \mathrm{IFs} .
$$

For the (total) c-boundary, the so-called S-relation comes into play [27. Denote $\hat{M}_{\emptyset}=\hat{M} \cup\{\emptyset\}$ (resp. $\left.\check{M}_{\emptyset}=\check{M} \cup\{\emptyset\}\right)$. The S-relation $\sim_{S}$ is defined in $\hat{M}_{\emptyset} \times \check{M}_{\emptyset}$ as follows. First, in the case $(P, F) \in \hat{M} \times \check{M}$,

$$
P \sim_{S} F \Longleftrightarrow\left\{\begin{array}{lll}
P & \text { is included and is a maximal IP into } & \downarrow F \\
F & \text { is included and is a maximal IF into } & \uparrow P .
\end{array}\right.
$$

By maximal we mean that no other $P^{\prime} \in \hat{M}$ (resp. $F^{\prime} \in \check{M}$ ) satisfies the stated property and includes strictly $P$ (resp. $F$ ). Recall that, as proved by Szabados [27, $I^{-}(p) \sim_{S} I^{+}(p)$ for all $p \in M$, and these are the unique S-relations (according to our definition (12)) involving proper indecomposable sets. Now, in the case $(P, F) \in \hat{M}_{\emptyset} \times \check{M}_{\emptyset} \backslash\{(\emptyset, \emptyset)\}$, we also put

$$
P \sim_{S} \emptyset, \quad\left(\text { resp. } \emptyset \sim_{S} F\right)
$$

if $P$ (resp. $F$ ) is a (non-empty, necessarily terminal) indecomposable past (resp. future) set that is not S-related by $(12)$ to any other indecomposable set; notice that $\emptyset$ is never S-related to itself. Now, we can introduce the notion of c-completion, according to $[9]$ :

Definition 4.2 Let $(M, g)$ be a strongly causal spacetime. The c-completion of $(M, g)$ is defined as follows. 
- As a point set: the c-completion $\bar{M}$ is formed by the pairs of TIPs and TIFs which are S-related, that is,

$$
\bar{M}:=\left\{(P, F) \in \hat{M}_{\emptyset} \times \check{M}_{\emptyset}: P \sim_{S} F\right\} .
$$

Every point $p \in M$ of the manifold will be identified with its corresponding pair $\left(I^{-}(p), I^{+}(p)\right)$, so $M$ will be considered a subset of $\bar{M}$ and, thus, the c-boundary is defined as $\partial M=\bar{M} \backslash M$.

- As a chronological set: two pairs $(P, F),\left(P^{\prime}, F^{\prime}\right) \in \bar{M}$ are chronologically related, denoted $(P, F) \ll\left(P^{\prime}, F^{\prime}\right)$, if $F \cap P^{\prime} \neq \emptyset$.

- Topologically: $\bar{M}$ is endowed with the chronological topology $\tau_{c h r}$, i.e., the sequential topology associated to the following limit operator (recall Definition 2.1):

$$
L_{c h r}(\sigma):=\left\{(P, F) \in \bar{M}: \begin{array}{l}
P \in \hat{L}\left(\left\{P_{n}\right\}\right) \text { if } P \neq \emptyset \\
F \in \check{L}\left(\left\{F_{n}\right\}\right) \text { if } F \neq \emptyset
\end{array}\right\} \quad \text { for any } \sigma=\left\{\left(P_{n}, F_{n}\right)\right\} \subset \bar{M},
$$

where

$$
\begin{aligned}
& P \in \hat{L}\left(\left\{P_{n}\right\}\right) \Longleftrightarrow P \subset \operatorname{LI}\left(\left\{P_{n}\right\}\right) \text { and it is maximal in } \operatorname{LS}\left(\left\{P_{n}\right\}\right) \\
& F \in \check{L}\left(\left\{F_{n}\right\}\right) \Longleftrightarrow F \subset \operatorname{LI}\left(\left\{F_{n}\right\}\right) \text { and it is maximal in } \operatorname{LS}\left(\left\{F_{n}\right\}\right) .
\end{aligned}
$$

The main properties of this choice of c-completion $\left(\bar{M}, \mathbb{\ll}, \tau_{c h r}\right)$ are summarized in the following result (see [9, Theorem 3.27]):

Theorem 4.3 The c-completion $\left(\bar{M}, \mathbb{\ll}, \tau_{c h r}\right)$ in Defn. 4.2 satisfies the following properties:

(i) Any future-directed (resp. past-directed) inextendible timelike curve in $M$ (namely, $\gamma$ : $[a, b) \rightarrow M)$ has an endpoint in $\partial M$.

(ii) The inclusion $i: M \hookrightarrow \bar{M}$ is a topological embedding and $i(M)$ is dense in $\bar{M}$.

(iii) The c-boundary $\partial M$ is closed in $\bar{M}$.

(iv) $I^{ \pm}((P, F))$ is open for any $(P, F) \in \bar{M}$.

(v) The topology $\tau_{c h r}$ is $T_{1}$.

\subsection{The modified c-boundary}

Given the limit operator $L_{c h r}$ and its associated chronological topology $\tau_{c h r}:=\tau_{L_{c h r}}$ on $\bar{M}$, we put $D=M$ and consider the minimally $M$-separating topology $\tau_{c h r}^{*}$, the minimally sequentially $M$-separating topology $\left(\tau_{c h r}^{*}\right)_{S e q}$, the operator $L_{c h r}^{*}$ and its associated topology $\tau_{L_{c h r}^{*}}$. In the beginning of Section 3 we imposed a "minimality" condition for our refinements in order to ensure that both, the original topology and the refined ones shared as many properties as possible (except the new properties). Therefore, it is expected that the refinements defined along such a section (summarized in Remark 3.10 and Figure 1) will also satisfy the properties included in Theorem 4.3 In fact, we can prove the following result:

Theorem 4.4 The topological spaces $\left(\bar{M}, \tau_{c h r}^{*}\right),\left(\bar{M},\left(\tau_{c h r}^{*}\right)_{\text {Seq }}\right)$ and $\left(\bar{M}, \tau_{L_{c h r}^{*}}\right)$ endowed with the chronological relation $\ll$ satisfy all the assertions (i)-(v) in Theorem 4.3. Moreover, the points on $M$ are $T_{2}$-separated from the points on $\partial M$ with the topologies $\tau_{c h r}^{*}$ and $\left(\tau_{c h r}^{*}\right)_{\text {Seq }}$, and the latter is equal to $\tau_{L_{c h r}^{*}}$ if $L_{c h r}$ is of first order. 
Proof. Assertions (iii), (iv) and (v) are straightforward as all topologies are finer than the chronological one, and this one satisfies them (by Theorem 4.3). Next, recall that, for any future (resp. past) inextendible timelike curve $\gamma:[a, b) \rightarrow M$ and any sequence $t_{n} \nearrow b$, the following equation

$$
\begin{gathered}
L\left(\left\{\gamma\left(t_{n}\right)\right\}\right)=\left\{(P, F) \in \partial M: P=I^{-}(\gamma)\right\} \\
\left(\text { resp. } L\left(\left\{\gamma\left(t_{n}\right)\right\}\right)=\left\{(P, F) \in \partial M: F=I^{+}(\gamma)\right\}\right)
\end{gathered}
$$

is true for both, $L_{c h r}$ and $L_{c h r}^{*}$. So, assertion (i) is implicitly proved in [9, Theorem 3.27], while the second part of (ii) is a consequence of the fact that no sequence $\left\{\gamma\left(t_{n}\right)\right\}$ with $t_{n} \nearrow b$ converges to a point in $M$. On the other hand, the first assertion in (ii) follows from the first assertion of Theorem 4.3 (ii) and the fact that $\left.L_{c h r}^{*}\right|_{M}=\left.L_{c h r}\right|_{M}$ (recall (8), i.e., formula (14) below and Proposition 2.7]. Finally, the last assertion is obtained from Theorem 3.9.

Remark 4.5 (1) The topology $\tau_{c h r}^{*}$ may not be Hausdorff, as two points at the boundary $\partial M$ may be non- $\mathrm{T}_{2}$-separated. However, such type of examples are natural in different situations. Namely, this happens if one removes a half time axis of Lorentz-Minkowski $\mathbb{L}^{2}$ (as the removed origin would yield naturally two boundary points), or in more refined examples such as the "grapefruit on a stick" in [7] or the two chimneys construction in [11, Appendix]. In these examples, the ideal points which are non- $\mathrm{T}_{2}$-separated for $\tau_{c h r}$ remain non- $\mathrm{T}_{2}$-separated for $\tau_{c h r}^{*}$ -but this can be regarded as harmless.

(2) Conditions (i)-(v) of both, Theorem 4.3 and Theorem 4.4 remain true with independence of the fact that $L_{c h r}$ or $L_{c h r}^{*}$ may or not be of first order. The first order condition is only necessary to ensure that $\tau_{L_{c h r}^{*}}$ satisfies $\left(\mathrm{A}_{\mathrm{Sep}}\right)$.

\section{Admissibility criteria for c-boundaries}

A strong support for the choice of the explained definition of c-completion in [9], is that such a choice follows from a set of minimal hypotheses, which catch the intuitive requirements that a c-boundary must fulfill. But, as pointed out in that reference, one could add more hypotheses if further properties were required for that boundary. Let us revisit first the original hypotheses, and then, let us add the minimal separability of the boundary as one of these hypotheses.

\subsection{Original criteria}

The admissibility conditions for the c-boundary in [9] provide the point set and chronological structures previously explained in subsection 4.2 Let us remind those for the topology.

Definition 5.1 Consider a strongly causal spacetime $M$ and its c-completion $\bar{M}$ regarded only as a point set and a chronological set (see Defn. 4.2). The following hypotheses are conditions of admissibility for a topology $\tau$ on $\bar{M}$ :

(A1) For all $(P, F) \in \bar{M}$, the sets $I^{ \pm}((P, F))$ are open.

(A2) The limits for $\tau$ are compatible with the empty set, i.e., if $\left\{\left(P_{n}, F_{n}\right)\right\}_{n} \rightarrow(P, \emptyset)$ (resp. $(\emptyset, F)$ ) and there exists $\left(P^{\prime}, F^{\prime}\right) \in \bar{M}$ such that $P \subset P^{\prime} \subset \operatorname{LI}\left(\left\{P_{n}\right\}\right)$ (resp. $F \subset F^{\prime} \subset$ $\left.\mathrm{LI}\left(\left\{F_{n}\right\}\right)\right)$, then $\left(P^{\prime}, F^{\prime}\right)=(P, \emptyset)$ (resp. $\left.\left(P^{\prime}, F^{\prime}\right)=(\emptyset, F)\right)$.

$\left.\overline{(A}_{\text {Min }}\right) \tau$ is minimally fine among the topologies satisfying previous conditions, i.e., no other topology satisfying the conditions (A1) and (A2) is strictly coarser than $\tau$. 
$\left(\bar{A}_{M i n}^{\text {Seq }}\right) \tau$ is minimally fine among the sequential topologies satisfying previous conditions (A1) and (A2), i.e., $\tau$ is sequential and no other sequential topology satisfying conditions (A1) and (A2) is strictly coarser than $\tau$.

A topology $\tau$ satisfying the conditions (A1), (A2) and $\left(\bar{A}_{\text {Min }}\right)$ will be called generally admissible and one satisfying (A1), (A2) and $\left(\bar{A}_{\text {Min }}^{\text {Seq }}\right)$ will be sequentially admissible or simply admissible. Accordingly, the c-completion $\bar{M}$ endowed with such a topology will be called generally admissible or admissible.

Remark 5.2 Here we have reserved the term "admissible topology" for the plain notion defined above, which agrees with [9] and subsequent references. When, in the spirit of [9, Sect. 3.1.3], further hypotheses are imposed, we will use a different name (as in Definition 5.6 below), in order to avoid confusions.

Let us discuss briefly the conditions in Definition 5.1 (see 9 for further details). Condition (A1) determines partially the convergence of sequences. In fact, for any topology $\tau$ satisfying (A1):

$$
\left(P_{n}, F_{n}\right) \rightarrow(P, F) \Rightarrow P \subset \operatorname{LI}\left(\left\{P_{n}\right\}\right), F \subset \operatorname{LI}\left(\left\{F_{n}\right\}\right) .
$$

Moreover, if, in addition, $P \neq \emptyset \neq F$, we have the following implication in terms of the operator $L_{c h r}$ of the chr-chronology:

$$
\left(P_{n}, F_{n}\right) \rightarrow(P, F) \Rightarrow(P, F) \in L_{c h r}\left(\left\{\left(P_{n}, F_{n}\right)\right\}\right) .
$$

(see [9, Lemma 3.15] for details). Condition (A2) is a compatibility requirement for the convergence of sequences when a component of the limit pair is empty. In particular, one can extend formula $\sqrt{13}$ to any $(P, F) \in \bar{M}$, that is, if a topology $\tau$ satisfies (A1) and (A2) and $\left(P_{n}, F_{n}\right) \rightarrow(P, F)$ one has:

$$
\begin{gathered}
\left(P_{n}, F_{n}\right) \rightarrow(P, \emptyset) \Rightarrow(P, \emptyset) \in L_{c h r}\left(\left\{\left(P_{n}, F_{n}\right)\right\}\right) \\
\left(\operatorname{resp.}\left(P_{n}, F_{n}\right) \rightarrow(\emptyset, F) \Rightarrow(\emptyset, F) \in L_{c h r}\left(\left\{\left(P_{n}, F_{n}\right)\right\}\right)\right) .
\end{gathered}
$$

(see [9, Prop. 3.20] for details). Summing up, the following property is obtained for referencing:

Lemma 5.3 If $\sigma \rightarrow(P, F)$ with any topology $\tau$ satisfying $(A 1)$, (A2) then $(P, F) \in L_{\text {chr }}(\sigma)$.

Finally, conditions $\left(\overline{\mathrm{A}}_{M i n}\right)$ or $\left(\overline{\mathrm{A}}_{M i n}^{\text {Seq }}\right)$ are minimality conditions which will allow to speak on uniqueness and guarantee that no spurious sets are included in the topology.

From the viewpoint of "first principles", the following result, which clarifies 9, Theorem 3.22], justifies the choice of the chronological topology for the c-completion (at least when $L_{c h r}$ is of first order).

Theorem 5.4 Let $\bar{M}$ be the c-completion of a strongly causal spacetime $M$ regarded as a point set and a chronological set. If the limit operator $L_{c h r}$ is of first order, then the chr-topology is the unique (sequentially) admissible topology on $\bar{M}$. Moreover, in this case, $L_{c h r}$ is also the associated limit operator of any generally admissible topology $\tau \subset \tau_{\text {chr }}$.

Proof. The first assertion is obtained in [9, Theorem 3.22], but we can sketch now a proof by using our previous results as follows. First, a straightforward computation shows that $L_{c h r}$ satisfies (A1) and (A2). By Lemma 5.3, the limit operator $L$ associated to any sequential topology obeying (A1) and (A2) must satisfy $L \subset L_{c h r}$, and the minimality assumption $\left(\overline{\mathrm{A}}_{M i n}^{\text {Seq }}\right)$ implies $L=L_{\text {chr }}$. 
For the last assertion, assume that there exists a generally admissible topology $\tau \subset \tau_{c h r}$ and denote by $L_{\tau}$ its associated limit operator. On the one hand, from Proposition 2.5 (b), we deduce that $\tau_{\text {Seq }} \subset \tau_{L_{c h r}}$ and, as $L_{\tau}$ is of first order, Lemma 2.4 ensures that $L_{c h r}(\sigma) \subset L_{\tau}(\sigma)$ for all sequence $\sigma \subset \bar{M}$. On the other hand, recall that the limit operator $L_{\tau}$ is associated to a topology $\tau$ fulfilling the admissibility conditions, and so, it must also obey $L_{\tau}(\sigma) \subset L_{c h r}(\sigma)$ for all sequence by Lemma 5.3 . In conclusion, $L_{\tau}=L_{c h r}$.

Remark 5.5 As the operator $L_{c h r}$ is not of first order only in very artificial cases (see Example 6.2 , this theorem (plus the properties of Theorem 4.3) gives a strong support for the usage of the chr-topology, at least if one does not care on the $T_{2}$-separability of the boundary. In fact, if a redefinition of the c-boundary topology preserved the expected good properties, then it would agree with the chr-topology whenever $L_{c h r}$ is of first order. Moreover, in this case the last assertion of previous theorem ensures that, whenever generally admissible topologies exist, they are minimally fine among the topologies with $L_{c h r}$ as an associated limit. When $L_{c h r}$ is not of first order then the chronological topology is not admissible (this can be checked directly from Lemma 5.3. However, the chronological topology would still make sense and we will explore even this case.

\subsection{Including $D$-separability as an admissibility condition}

As shown in Section 3 the $T_{2}$-separation of points of $M$ and $\partial M$ can be always obtained as a requirement a posteriori. However, one can think that such a property should be included as one of the a priori properties of the c-boundary, at the same level of the other admissibility conditions. Next, this approach is developed.

Recall first that the limit operator of the searched ( $T_{2}$-separating) topology $\tau$ must satisfy also the admissibility conditions (A1) and (A2) and, so, Lemma 5.3 implies $L_{\tau} \subset L_{c h r}$. We focus on the case of sequential topologies because, on the one hand, the topology of the c-boundary is studied by considering the convergence of some sequences and, on the other, results such as Theorem 5.4 show that they are specially interesting (of course, this can be extended to non-sequential ones, as we have done till now).

Definition 5.6 A sequential topology $\tau$ is $T_{2}$-admissible for the c-completion $\bar{M}$ if it satisfies properties (A1) and (A2) of Definition 5.1, together with $\left(A_{S e p}\right)$ in Definition 3.1 and the following one:

$\left(\tilde{A}_{M i n}^{S e q}\right) \tau$ is minimally fine among the sequential topologies satisfying previous conditions, i.e., no other sequential topology satisfying (A1), (A2) and ( $\left.A_{S e p}\right)$ is strictly coarser than $\tau$.

Taking into account Proposition 3.7, we consider the operator

$$
L_{c h r}^{*}(\sigma):=\left\{\begin{array}{l}
L_{c h r}(\sigma) \cap M \\
L_{c h r}(\sigma)
\end{array} \quad \text { if } \exists \kappa \subset \sigma \text { and } p \in M \text { such that } p \in L_{c h r}(\kappa)\right.
$$

consistently with (8). If we apply Theorem 3.9 (ii) to $\left(\bar{M}, \tau_{c h r}\right)$ and $D=M$, we deduce directly:

Corollary 5.7 Let $\bar{M}$ be the c-completion of a strongly causal spacetime $M$ regarded as a point set and a chronological set. If the limit operator $L_{c h r}$ is of first order, then the operator $L_{c h r}^{*}$ is also of first order, and its associated topology $\tau_{L_{c h r}^{*}}$ is equal to $\left(\tau_{c h r}^{*}\right)_{\text {Seq }}$, i.e., $\tau_{L_{c h r}^{*}}$ is the unique sequentially minimally D-separating topology for $D=M$. 
However, one can go a step further in order to check that $\tau_{L_{c h r}^{*}}$ is the suitable topology for the requirements in Definition 5.6 (in the same sense that Theorem 5.4 proved that $\tau_{L_{c h r}}$ was the suitable topology for Definition 5.1) by imposing directly the first order character to $L_{c h r}^{*}$.

Theorem 5.8 Let $\bar{M}$ be the c-completion of a strongly causal spacetime $M$ regarded as a point set and a chronological set. If $L_{c h r}^{*}$ is of first order on $\bar{M}$, then the topology $\tau_{L_{c h r}^{*}}$ satisfies (A1), (A2) and $\left(A_{S e p}\right)$. If, in addition, $L_{c h r}$ is of $k$-th order for some ordinal $k$ (in particular, both hypotheses hold when $L_{c h r}$ is of first order), then $\tau_{L_{c h r}^{*}}$ also satisfies $\left(\tilde{A}_{M i n}^{\text {Seq }}\right)$, i.e., $\tau_{L_{c h r}^{*}}$ is the unique $T_{2}$-admissible sequential topology of $\bar{M}$.

Proof. Let us begin with the first assertion, that is, let us show that $\tau_{L_{c h r}^{*}}$ satisfies (A1), (A2) and $\left(\mathrm{A}_{\mathrm{Sep}}\right)$ if $L_{c h r}^{*}$ is of first order. Since $L_{c h r}^{*} \subset L_{c h r}$, thus $\tau_{c h r} \subset \tau_{L_{c h r}^{*}}$ (see Lemma 2.4), and $\tau_{c h r}$ satisfies (A1), we deduce that $\tau_{c h r}^{*}$ also satisfies (A1). Moreover, from Proposition 3.7 and Lemma 3.6, we know that $\tau_{L_{c h r}^{*}}$ satisfies $\left(\mathrm{A}_{\mathrm{Sep}}\right)$. In order to prove (A2), assume that $\sigma=\left\{\left(P_{n}, F_{n}\right)\right\} \rightarrow(P, \emptyset), P \neq \emptyset$, with $\tau_{c h r}^{*}$ (the case $P=\emptyset \neq F$ is analogous) and assume the existence of $\left(P^{\prime}, F^{\prime}\right) \in \bar{M}$ such that $P \subset P^{\prime} \subset \operatorname{LI}\left(\left\{P_{n}\right\}\right)$. As $L_{c h r}^{*}$ is of first order on $\bar{M}$, $(P, \emptyset) \in L_{c h r}^{*}(\sigma)$. Taking into account that $(P, \emptyset) \in \partial M$, necessarily $L_{c h r}^{*}(\sigma)=L_{c h r}(\sigma)$, and thus, $(P, \emptyset) \in L_{c h r}(\sigma)$. In particular, $P$ is a maximal IP in $\operatorname{LS}\left(\left\{P_{n}\right\}\right)$. Therefore, as $\left(P^{\prime}, F^{\prime}\right) \in \bar{M}$ satisfies $P \subset P^{\prime} \subset \operatorname{LI}\left(\left\{P_{n}\right\}\right)$, necessarily $P=P^{\prime}$ and $F^{\prime}=\emptyset$, as required.

For the second assertion, assume additionally that $L_{c h r}$ is of $k$-th order and let us show that $\tau_{L_{c h r}^{*}}$ also satisfies $\left(\tilde{\mathrm{A}}_{\mathrm{Min}}^{S e q}\right)$. In fact, we are going to prove something even stronger, concretely, that any sequential topology $\tau_{L}$ satisfying (A1), (A2) and (A $\left.\mathrm{A}_{\mathrm{Sep}}\right)$, must obey $\tau_{L_{c h r}^{*}} \subset \tau_{L}$. On the one hand, as $L_{c h r}$ is of $k$-th order, Proposition 3.12 ensures that $L_{c h r}^{*}=L_{\tau_{c h r}}^{*}$, and so, from Theorem 3.9 (ii), we deduce that $\tau_{L_{c h r}^{*}}$ is the unique sequentially minimally $D$-separating topology. On the other hand, as $\tau_{L}$ satisfies (A1) and (A2), Lemma 5.3 ensures that $L(\sigma) \subset L_{c h r}(\sigma)$ for all sequence $\sigma$ and so, $\tau_{c h r} \subset \tau_{L}$, i.e., $\tau_{L}$ satisfies $\left(\mathrm{A}_{\text {Fin }}\right)$. Taking into account that $\tau_{L}$ also satisfies $\left(\mathrm{A}_{\mathrm{Sep}}\right)$ and the definition of sequentially minimally $D$-separating topology, we deduce that $\tau_{L_{c h r}^{*}} \subset \tau_{L}$, as desired.

Figure 3 summarizes the results of this section, for the convenience of the reader.

Remark 5.9 As the hypotheses of Theorem 5.8 are less restrictive than the first order property for $L_{c h r}$, its conclusion is sharper than Corollary 5.7 (see Example 6.2). But, beyond this subtlety, either Theorem 5.8 or Corollary 5.7 show that no matter if the $T_{2}$-separability is imposed a priori $\left(T_{2}\right.$-admissible) or a posteriori ( $D$-separating $)$.

Summing up, the question of $T_{2}$-separability can be circumvented for the c-boundary. From a fundamental viewpoint, one can work with the $T_{2}$-separating topology $\tau_{L_{c h r}^{*}}$, as the admissibility properties of this topology yield more accurate consequences than those for $\tau_{L_{c h r}}$ (Corollary 5.7 , Theorem 5.8). In any case, as pointed out in Remark 5.5, the cases where the topologies $\tau_{c h r}$ or $\tau_{L_{c h r}^{*}}$ satisfy the conditions of admissibility are so general that one would not be especially worried with the cases where they differ. And, from the practical viewpoint, this way of proceeding is equivalent to consider the chr-completion (as defined in [9]) and impose the $T_{2}$-separation of $M$ and $\partial M$, as one can do in the general framework of Section 3 Nevertheless, if further studies suggested that more separability properties must be required for the c-boundary, one could remake our previous process by including such properties, in the spirit of [9].

\section{Appendix: some examples}

This section is devoted to present some examples in order to illustrate some of the assertions appeared in previous sections. The first two examples were already studied in [8] and [1], resp., 


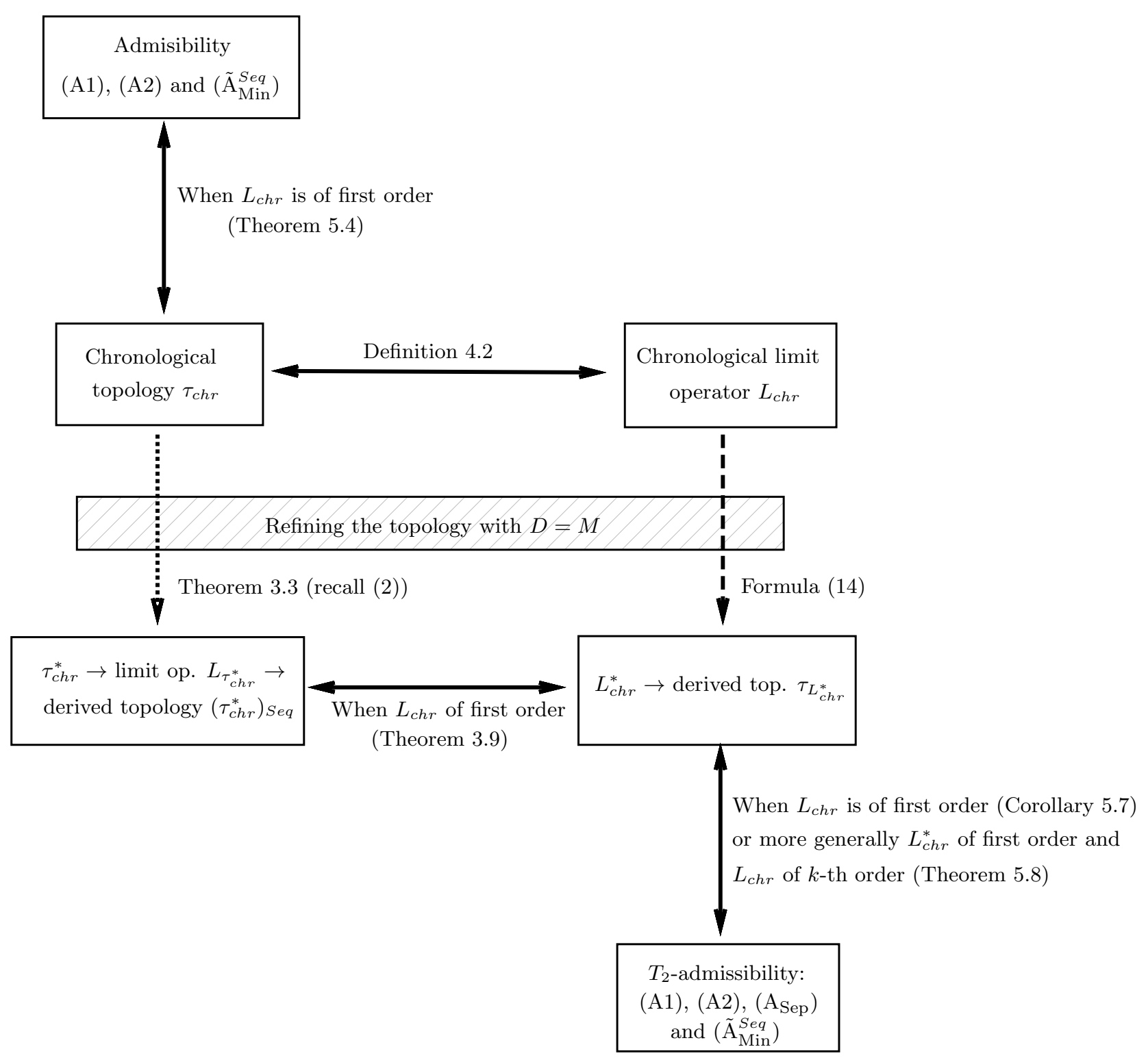

Figure 3: This figure summarizes the main results in Section 5 .

Recall that Section 3 provides two different ways to refine the topology $\tau_{c h r}$ in order to obtain the separability condition on $D=M$ : (a) focusing on the chronological topology itself (following the dotted arrow) or (b) considering the limit operator $L_{c h r}$ (following the dashed arrow). When $L_{c h r}^{*}$ is of first order the procedure (b) is equivalent to include $T_{2}$-separability as an admissibility condition for the c-boundary (Theorem 5.8). In any case, when $L_{c h r}$ is of first order (which is slightly more restrictive than $L_{c h r}^{*}$ of first order), the procedures (a) and (b) are equivalent (Theorem 3.9. 


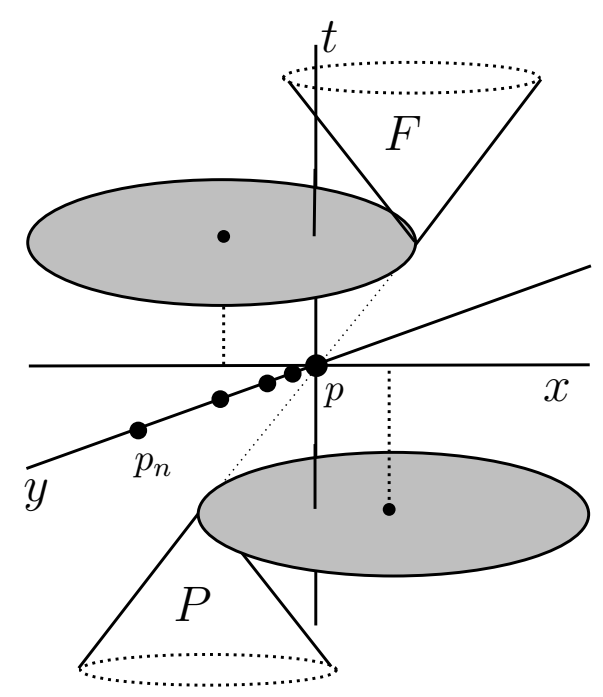

Figure 4: Minkowski spacetime $\mathbb{L}^{3}$ with the dashed regions (two discs of radius 2 centered at the points $(-1,0,1)$ and $(1,0,-1))$ removed.

so here we will just summarize the key points.

Example 6.1 Let us begin by showing the possibility for the c-boundary of the setting that motivates this paper, that is, the existence of a c-completion $\bar{M}$ where two points, one on the manifold and other on the boundary, are not $\mathrm{T}_{2}$-related. For this, we will just recall the two examples introduced in [8, Section 2.3]. Such examples show not only that the c-completion may present separability problems, but also that the involved boundary points can be represented by any type of pairs $(P, F)$ (i.e., with both or just one non-empty component).

On the first example (represented in Figure 4), the non-empty sets $P$ and $F$ are S-related, and so, they determine a point at the boundary. Moreover, from the definition of the chronological limit (recall Def. 4.2), we have that the sequence $\left\{p_{n}\right\}$ converges to both, the manifold point $p \in M$ and the boundary point $(P, F) \in \partial M$.

On the second example (Figures 5 and 6 ), there are two boundary points attached to $q$. In fact, the set $P$ (which includes $P^{\prime}$ but it is indecomposable) is S-related with $F$ while the set $P^{\prime}$ is S-related with the empty set, obtaining the boundary points $(P, F),\left(P^{\prime}, \emptyset\right) \in \partial M$. Here, the sequence $\left\{p_{n}\right\}$ converges again to both, a point in the manifold $p \in M$, and a boundary point having one empty component $\left(P^{\prime}, \emptyset\right) \in \partial M$.

Example 6.2 We are going to show that the limit operator $L_{c h r}$ of the completion of a spacetime is not necessarily of first order (suggesting a procedure for the construction of examples of $k$-th order for any $k \in \mathbb{N} \cup\left\{\aleph_{0}\right\}$ ). To this aim, we explain first a key example which is described with further details in [1, Chapter 4].

The spacetime $M$ consists of $\mathbb{L}^{3}$ with the following subsets removed (see Figures 7, 8): the causal future $J^{+}(0,0,0)$, the sheets $\left\{\Pi_{n}^{\infty}\right\}_{n \geq 1}$, where $\Pi_{n}^{\infty}=J^{-}(0,0,0) \cap \Pi_{n}$ and $\Pi_{n}$ is the plane $t-1 / n=0$, the semi-discs $\left\{D_{n}\right\}_{n \geq 1} \cup D_{\infty}$ and the sheets $\left\{\Pi_{n}^{l}\right\}_{l, n \geq 1}$ (obtained from $\left\{\Pi_{n}^{\infty}\right\}_{n}$ by a convenient "contraction and translation"). The spacetime $M$ presents a number of distinguished indecomposable sets, which are also depicted in the figures, say: $P_{\infty}, P_{\infty}^{\prime}, F=I^{+}\left(p_{0}\right)$ and 


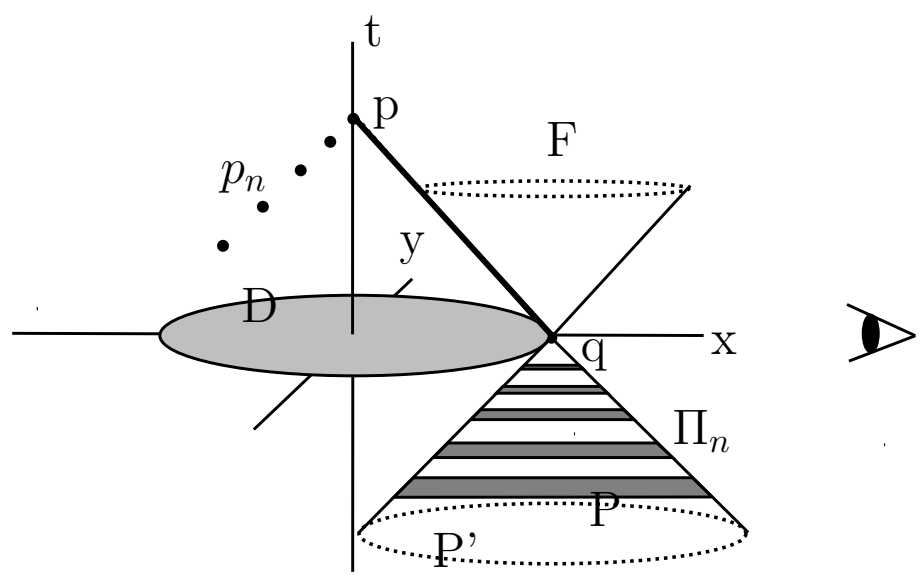

Figure 5: Representation of the spacetime $M$, obtained by removing from $\mathbb{L}^{3}$ the dashed regions, which consist of the unitary disc $D$ and the sheets $\Pi_{n}$ (see Figure 6 for a lateral view of the sheets, which allows to distinguish between $P$ and $P^{\prime}$ ).

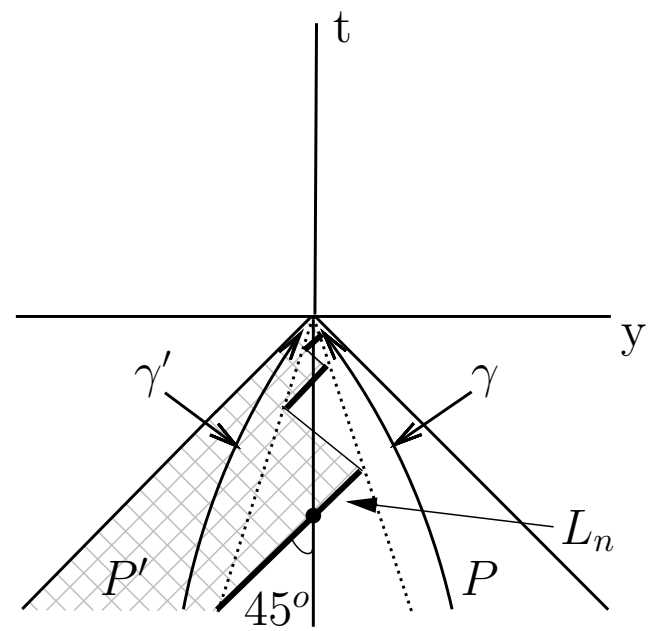

Figure 6: Slice of $M$ at $x=x_{0}$, as seen from the "eye" in previous figure. The segments $L_{n}=$ $\pi_{n} \cap\left\{x=x_{0}\right\}$ for some $x_{0} \in \mathbb{R}$ are represented. The curves $\gamma$ and $\gamma^{\prime}$ which defines $P$ and $P^{\prime}$ as IPs are here also depicted. In fact, $P=I^{-}(\gamma)$ includes the region between the two half lines at \pm 45 degrees with respect to the axis, while $P^{\prime}=I^{-}\left(\gamma^{\prime}\right)$ is only the subregion striped with thin lines at \pm 45 degrees. 


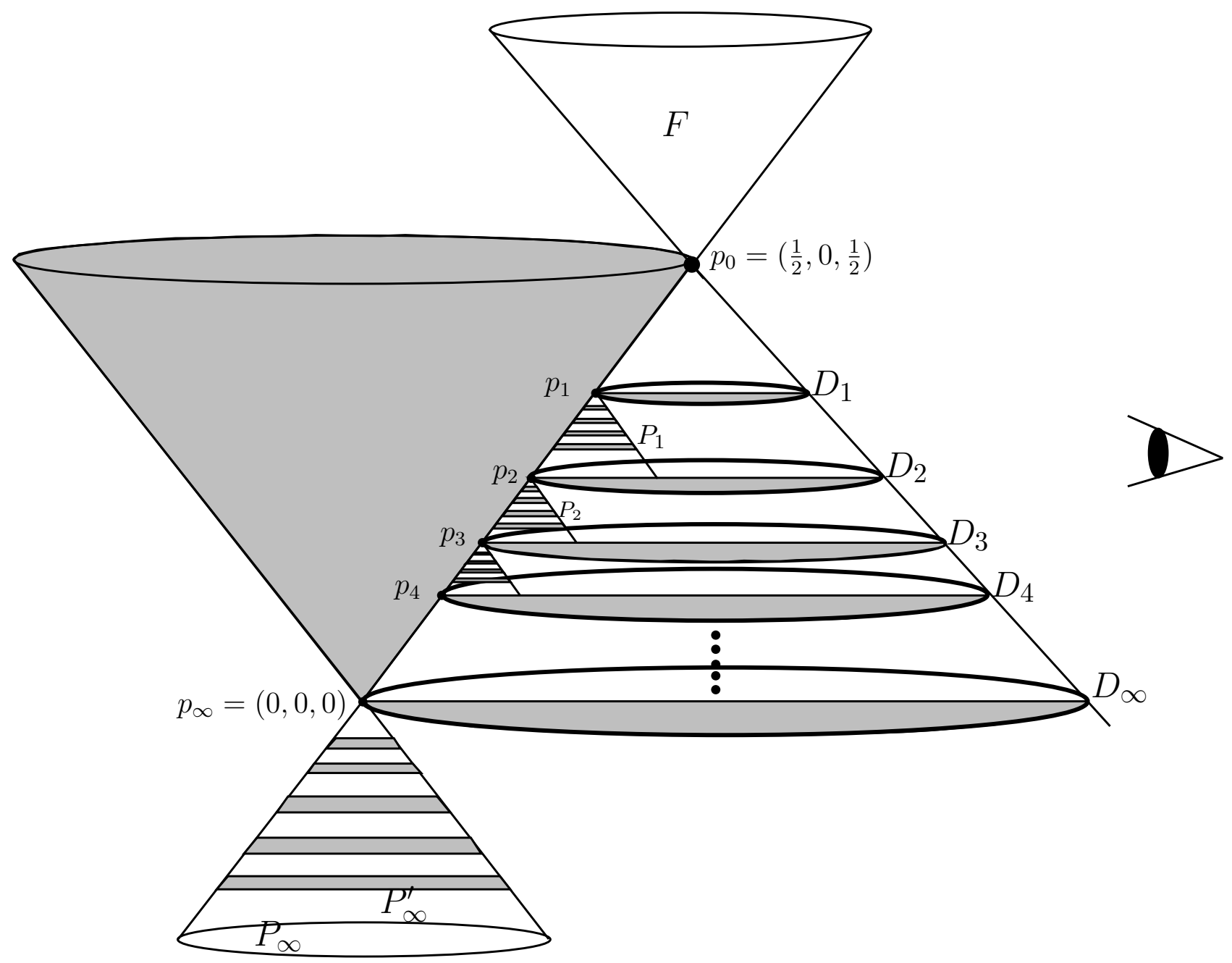

Figure 7: Representation of the spacetime $M$, obtained by removing from $\mathbb{L}^{3}$ the grey regions. The figure also shows the distinguished IPs, $P_{\infty}, P_{\infty}^{\prime}$ and $\left\{P_{n}\right\}_{n \geq 1}$ (see Figure 8). Observe that every $P_{n}$ and $P_{n}^{\prime}$ consists of a "contraction plus a translation" of $P_{\infty}$ and $P_{\infty}^{\prime}$ resp.

$P_{n}, P_{n}^{\prime}$ with $n \geq 1$; notice that each $\left(P_{n}, F\right)$ (rather than $\left.p_{n}\right)$ as well as $\left(P_{\infty}, F\right)$ and $\left(P_{\infty}^{\prime}, \emptyset\right)$ are c-boundary points.

Consider the sequence $\sigma=\left\{x_{n}\right\} \subset M$, with $x_{n}=(1 / 2,1 / n, 1 / 2)$ for all $n$. Then, reasoning as in Example 6.1, $L_{c h r}(\sigma)$ contains $\left\{\left(P_{n}, F\right)\right\}_{n \geq 1}$ and $\left(P_{\infty}, F\right)$. Moreover, $\left(P_{\infty}^{\prime}, \emptyset\right) \in$ $L_{c h r}\left(\left\{\left(P_{n}, F\right)\right\}\right)$. So, $\sigma$ converges to $\left(P_{\infty}^{\prime}, \emptyset\right)$ with the chronological topology, as $\left(P_{\infty}^{\prime}, \emptyset\right) \in L_{\text {chr }}^{2}(\sigma)$ (recall (5) and Proposition 2.8). Note, however, that $\left.P_{\infty}^{\prime} \subsetneq P_{\infty} \subset L I\left(\left\{I^{-}\left(x_{n}\right)\right\}\right)\right)$. Hence, $P_{\infty}^{\prime}$ is not a maximal IP in $L S\left(\left\{I^{-}\left(x_{n}\right)\right\}\right)$, and thus, $\left(P_{\infty}^{\prime}, \emptyset\right) \notin L_{c h r}(\sigma)$. In conclusion, $L_{c h r}$ is not of first order. However, it seems that all the possible limits are contained in $L_{c h r}^{2}$ (and making a straightforward modification of the example it would be contained in some $L_{c h r}^{k}$ ), so it is conceivable the existence of examples where $L_{c h r}$ is a $k$-th order limit operator for any $k \in \mathbb{N} \cup\left\{\aleph_{0}\right\}$.

Example 6.3 Here, we present an example clarifying our definition of condition $\left(\mathrm{A}_{\mathrm{Sep}}\right)$. Concretely, we will justify the requirement that the open set $U$ belongs to the original topology $\tau_{M}$ of the manifold $M$ and not to the topology to be obtained. Basically, the reason of our choice is that the alternative topology might not preserve the original topology of the manifold $M$, as the following example shows.

Consider the spacetime $M$ depicted in Figure 5. It is not difficult to check that the chronological 


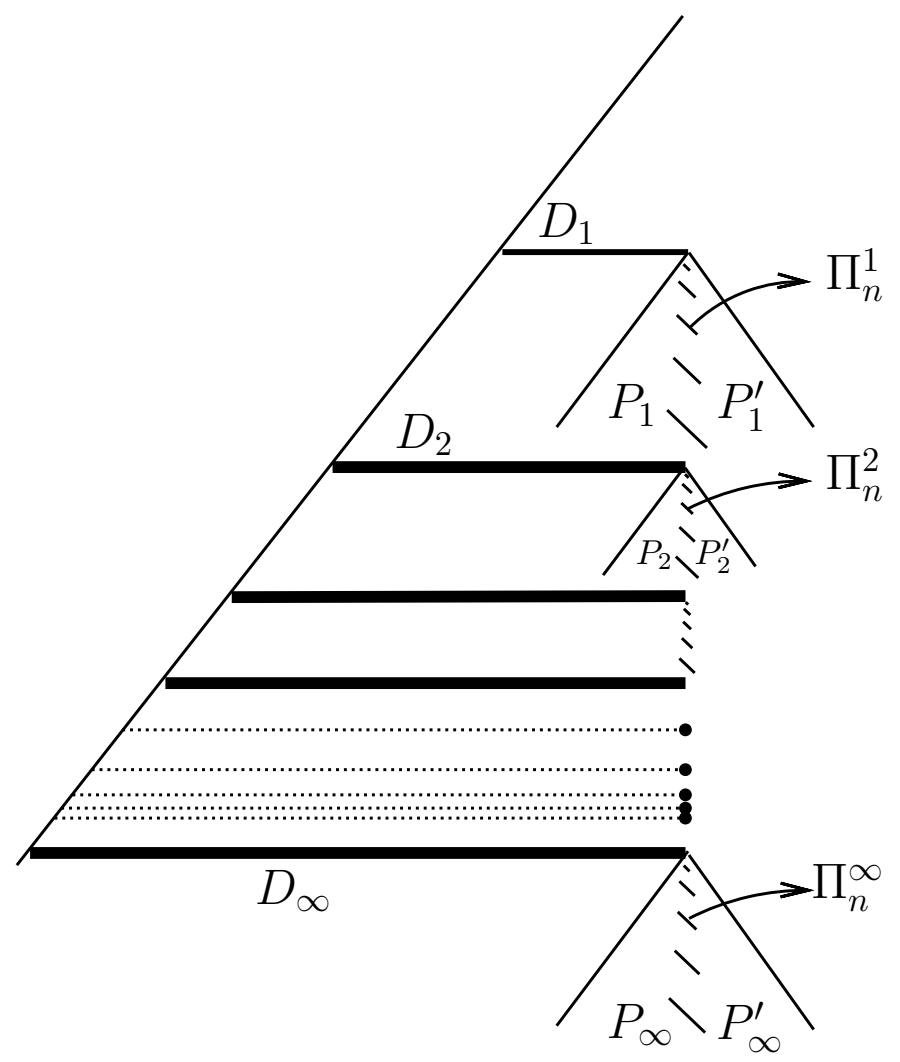

Figure 8: Section of $M$ as seen from the "eye" in previous figure. Note that, between two semi-discs $D_{i}$ and $D_{i+1}$, we have the same effect as in Example 6.1 (compare with Figures 5 and 6). Moreover, for all $n, P_{\infty} \not \subset P_{n}$ (in fact, $P_{m} \not \subset P_{n}$ for $m>n$ ), but $P_{\infty}^{\prime} \subset P_{n}$. This implies $P_{\infty}^{\prime} \subset L I\left(\left\{P_{n}\right\}\right)$ and $P_{\infty}^{\prime}$ is maximal IP in $L S\left(\left\{P_{n}\right\}\right)$, that is, $\left(P_{\infty}^{\prime}, \emptyset\right) \in L_{c h r}\left(\left\{\left(P_{n}, F\right)\right\}\right)$. 
limit operator $L_{c h r}$ is of first order. Let us define a new limit operator $L_{c h r}^{* *}$ as follows (compare with (8)):

$$
L_{c h r}^{* *}(\sigma):=\left\{\begin{array}{l}
L_{c h r}(\sigma) \cap \partial M \quad \text { if } \exists p \in M \text { and }(P, F) \in \partial M \text { such that } p,(P, F) \in L_{c h r}(\sigma) \\
L_{c h r}(\sigma) \\
\text { otherwise, }
\end{array}\right.
$$

and denote by $\tau_{c h r}^{* *}$ the topology associated to this limit operator. Since $L_{c h r}$ is of first order, it follows, by the same arguments as in Section 3.3, that $L_{c h r}^{* *}$ is a first order limit operator (recall Remark 5.9p. Moreover, its associated topology $\tau_{c h r}^{* *}$ is finer than $\tau_{c h r}, \mathrm{~T}_{2}$-separates the points of the boundary from the points of the manifold, and it is minimally finer among the sequential ones satisfying such properties. However, the sequence $\sigma=\left\{p_{n}\right\}$ in Figure 5 does not converge to $p$ with $\tau_{c h r}^{* *}$, since it satisfies $p,\left(P^{\prime}, \emptyset\right) \in L_{c h r}(\sigma)$ and so, $p \notin L_{c h r}^{* *}(\sigma)$. Taking into account that $\sigma$ clearly converges to $p$ with the manifold topology, we deduce that $\tau_{c h r}^{* *}$ does not preserves the manifold topology on $M$.

\section{Acknowledgments}

The authors are partially supported by the Spanish Grants MTM2013-47828-C2-1-P and MTM201347828-C2-2-P (MINECO and FEDER funds). The second-named author is also supported by FAPESP (Fundação de Amparo á Pesquisa do Estado de São Paulo, Brazil) Process 2012/119507.

\section{References}

[1] L. Aké: Sobre el borde causal en espacio-tiempos fuertemente causales, Tesis de Maestría, Universidad Autónoma de Yucatán, 2012.

[2] A.M. Amores; M. Gutiérrez, Construction of examples of b-completion. Nonlinear Anal. 47 (2001), no. 5, 2959-2970.

[3] R.A. Barry; S.M. Scott, The attached point topology of the abstract boundary for spacetime, Class. Quant. Grav. 28 (2011) 165003.

[4] R. A. Barry; S. M. Scott, The strongly attached point topology of the abstract boundary for space-time, Class. Quant. Grav. 31 (2014), 125004.

[5] B. Bosshard, On the b-boundary of the closed Friedmann-model, Comm. Math. Phys. 46 (1976), no. 3, 263-268.

[6] E. Caponio, M. A. Javaloyes, and M. Sánchez: On the interplay between Lorentzian causality and Finsler metrics of Randers type, Rev. Mat. Iberoamericana, 27 (2011), pp. 919-952.

[7] J.L. Flores, S.G. Harris: Topology of the causal boundary for standard static spacetimes, Class. Quant. Grav. 24 (5) (2007), 1211-1260.

[8] J.L. Flores, J. Herrera: The c-boundary construction of spacetimes: applications to stationary Kerr spacetime, Springer Proceedings in Mathematics $\&$ Statistics, 26 (2013), 243-275.

[9] J.L. Flores, J. Herrera, M. Sánchez: On the final definition of the causal boundary and its relation with the conformal boundary, Adv. Theor. Math. Phys. 15 (2011) 991-1058. 
[10] J.L. Flores, J. Herrera, M. Sánchez: Isocausal spacetimes may have different causal boundaries. Classical Quantum Gravity 28 (2011), no. 17, 175016, 9 pp.

[11] J.L. Flores, J. Herrera, M. Sánchez: Gromov, Cauchy and causal boundaries for Riemannian, Finslerian and Lorentzian manifolds, Mem. Amer. Math. Soc. 226 (2013), no. 1064, vi+76 pp.

[12] S. P. Franklin, Spaces in which sequences suffice, Fundamenta Mathematicae, volume 57, (1965), pp. 107-115

[13] R.P. Geroch, Local characterization of singularities in general relativity, J. Math. Phys. 9 (1968), 450-465.

[14] R.P. Geroch, E.H. Kronheimer, R. Penrose: Ideal points in spacetime, Proc. Roy. Soc. Lond. Ser. A 237 (1972), 545-567.

[15] R.P. Geroch, C.B Liang and R.M Wald, Singular boundaries of space-times, J. Math. Phys. 23(3) (1982), 432-435.

[16] A. Goreham: Sequential convergence in topological spaces (2004). Available at arXiv: math/0412558v1.

[17] M. Gutiérrez, Equivalence of Cauchy singular boundary and b-boundary in O(3)-reducible space-times, J. Geom. Phys. 59 (2009), no. 8, 1196-1198.

[18] S.G. Harris, Universality of the future chronological boundary, J. Math. Phys. 39(10) (1998), $5427-5445$.

[19] S.G. Harris, Topology of the future chronological boundary: universality for spacelike boundaries, Classical Quantum Gravity 17(3) (2000), 551-603.

[20] S.G. Harris, Boundaries on spacetimes: causality, topology, and group actions, Geom. Dedicata 126 (2007), 255-274.

[21] S.W. Hawking and G.F.R. Ellis, The large scale structure of space-time, Cambridge University Press, Cambridge, 1973.

[22] R.A. Johnson, The bundle boundary in some special cases, J. Mathematical Phys. 18 (1977), no. $5,898-902$.

[23] J. Margalef-Bentabol and E.J.S. Villaseñor, Topology of the Misner Space and its g-boundary, Gen. Relativ. Gravit. 46 (2014), 1755.

[24] S. Scott and P. Szekeres, The abstract boundary - a new approach to singularities of manifolds, J. Geom. Phys. 13(3) (1994), 223-253.

[25] B.G. Schmidt, A new definition of singular points in general relativity, Gen. Relativity Gravitation 1(3) (1970/71), 269-280.

[26] B.G. Schmidt, Remarks about modifications of the b-boundary definition, Gen. Relativity Gravitation 10(12) (1979), 981-982.

[27] L.B. Szabados: Causal boundary for strongly causal spaces, Class. Quant. Grav. 5 (1988), $121-134$.

[28] R.M. Wald, General relativity, The University of Chicago Press, Chicago, 1984. 